\title{
Targeted acetylation of NF-kappaB/RelA and histones by epigenetic drugs reduces post-ischemic brain injury in mice with an extended therapeutic window
}

\author{
Annamaria Lanzillotta ${ }^{\mathrm{a}, 1}$, Giuseppe Pignataro b, ${ }^{\text {, Caterina Branca }}{ }^{\text {a }}$, Ornella Cuomo ${ }^{\text {, }}$, Ilenia Sarnico ${ }^{\text {a }}$, \\ Marina Benarese ${ }^{\mathrm{a}}$, Lucio Annunziato ${ }^{\mathrm{b}, \mathrm{d}}$, PierFranco Spano ${ }^{\mathrm{a}, \mathrm{c}}$, Marina Pizzi ${ }^{\mathrm{a}, \mathrm{c}, *}$

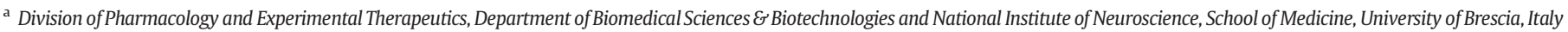 \\ b Division of Pharmacology, Department of Neuroscience and National Institute of Neuroscience, School of Medicine, Federico II University of Naples, Italy \\ c IRCCS, S. Camillo Hospital, Venice, Italy \\ d IRCCS SDN, Naples, Italy
}

\section{A R T I C L E I N F O}

\section{Article history:}

Received 3 April 2012

Revised 1 August 2012

Accepted 22 August 2012

Available online 30 August 2012

\section{Keywords:}

RelA acetylation

MCAO

OGD

HDAC inhibitors

\begin{abstract}
A B S T R A C T
Nuclear factor-kappaB (NF-kB) p50/RelA is a key molecule with a dual effect in the progression of ischemic stroke. In harmful ischemia, but not in preconditioning insult, neurotoxic activation of p50/RelA is characterized by RelA-specific acetylation at Lys310 (K310) and deacetylation at other Lys residues. The derangement of RelA acetylation is associated with activation of Bim promoter.

Objective: With the aim of producing neuroprotection by correcting altered acetylation of RelA in brain ischemia, we combined the pharmacological inhibition of histone deacetylase (HDAC) 1-3, the enzymes known to reduce global RelA acetylation, and the activation of sirtuin 1, endowed with a specific deacetylase activity on the K310 residue of RelA. To afford this aim, we tested the clinically used HDAC 1-3 inhibitor entinostat (MS-275) and the sirtuin 1 activator resveratrol.

Methods: We used the mouse model of transient middle cerebral artery occlusion (MCAO) and primary cortical neurons exposed to oxygen glucose deprivation (OGD).

Results: The combined use of MS-275 and resveratrol, by restoring normal RelA acetylation, elicited a synergistic neuroprotection in neurons exposed to OGD. This effect correlated with MS-275 capability to increase total RelA acetylation and resveratrol capability to reduce RelA K310 acetylation through the activation of an AMPactivated protein kinase-sirtuin 1 pathway. The synergistic treatment reproduced the acetylation state of RelA peculiar of preconditioning ischemia. Neurons exposed to the combined drugs totally recovered the optimal histone $\mathrm{H} 3$ acetylation.

Neuroprotection was reproduced in mice subjected to MCAO and treated with MS-275 (20 $\mu \mathrm{g} / \mathrm{kg}$ and $200 \mu \mathrm{g} / \mathrm{kg}$ ) or resveratrol $(6800 \mu \mathrm{g} / \mathrm{kg})$ individually. However, the administration of lowest doses of MS-275 $(2 \mu \mathrm{g} / \mathrm{kg})$ and resveratrol $(68 \mu \mathrm{g} / \mathrm{kg})$ synergistically reduced infarct volume and neurological deficits. Importantly, the treatment was effective even when administered $7 \mathrm{~h}$ after the stroke onset. Chromatin immunoprecipitation analysis of cortices harvested from treated mice showed that the RelA binding and histone acetylation increased at the $B C l-x_{L}$ promoter and decreased at the Bim promoter.

Conclusion: Our study reveals that epigenetic therapy shaping acetylation of both RelA and histones may be a promising strategy to limit post-ischemic injury with an extended therapeutic window.
\end{abstract}

(c) 2012 Elsevier Inc. All rights reserved.
Abbreviations: CBP, p300/CREB-binding protein; CSF, cerebrospinal fluid; DIV, days in vitro; LDH, lactate dehydrogenase; ip, intraperitoneal; K310, lysine 310; MCAO, middle cerebral artery occlusion; NF- $\kappa$ B, nuclear factor kappa B; OGD, oxygen glucose deprivation; SIRT1, sirtuin 1; HDAC, histone deacetylase; HAT, histone acetyltransferase; T172, threonine 172.

* Corresponding author at: Division of Pharmacology, Department of Biomedical Sciences \& Biotechnologies, Viale Europa, 11, 25123 Brescia, Italy. Fax: + 39030 3717529.

E-mail address: pizzi@med.unibs.it (M. Pizzi).

1 These authors contributed equally.

Available online on ScienceDirect (www.sciencedirect.com).

\section{Introduction}

Although advances in neurobiology outline a new era for brain restoration, total recovery of function following a stroke remains a rare accomplishment (Lloyd-Jones et al., 2010). The only therapeutic agent actually approved is the recombinant tissue plasminogen activator (tPA) within $3 \mathrm{~h}$ of symptom onset; however, less than $5 \%$ of stroke patients can benefit from the thrombolytic approach (Fang et al., 2010; Lees et al., 2010). Thus, identifying molecular targets for interventions that can be therapeutically implemented after stroke represents a compelling task for research in neurology. The mechanisms of neurodegeneration more involved in brain ischemia include excitotoxicity, calcium overload, 
oxidative stress, acute inflammation and apoptosis (Bramlett and Dietrich, 2004; Leker et al., 2002). Recently, defects in histone acetylation homeostasis have been recognized as yet another common feature in diverse neuropathological states including ischemic stroke (Hahnen et al., 2008). Acetylation is the key post-translational modification of histones that controls the accessibility of chromatin to the transcriptional machinery and plays an essential role in gene activation (Sweatt, 2009). The acetylation of lysines is reversible and controlled by the opposing actions of histone acetyltransferase (HAT) and histone deacetylase (HDAC). As over-deacetylation in brain ischemia is associated with a global decrease in HAT activity (Jin et al., 2001; Rouaux et al., 2003), HDAC inhibitors were evaluated and showed a therapeutic efficacy in mice models of middle cerebral artery occlusion (MCAO) (Faraco et al., 2006; Kim et al., 2007, 2009; Langley et al., 2008; Shein and Shohami, 2011). However, in the majority of studies conducted thus far, HDAC inhibitors were administered prior to or immediately following the onset of MCAO, a therapeutic intervention that is not feasible when treating stroke-inflicted individuals.

Besides histones, diverse non-histone proteins, including transcription factors NF-kB, are modified by HATs and HDACs (Haberland et al., 2009). Members of class I HDACs, particularly HDAC1, HDAC2, and HDAC3, are responsible for the general deacetylation of NF- $\mathrm{KB} / \mathrm{RelA}$ at five lysine residues (K122, 123, 218, 221, and 310) (Ashburner et al., 2001; Chen and Greene, 2004), while sirtuin 1, a class III HDAC activated by resveratrol, selectively deacetylates RelA at K310 (Lanzillotta et al., 2010; Yeung et al., 2004). Recent studies demonstrate that pro-apoptotic transcription induced by NF-KB p50/RelA in acute brain ischemia (Inta et al., 2006; Sarnico et al., 2009) relies on the specific derangement of normal RelA acetylation, i.e., the reduced level of total RelA acetylation despite an aberrant increase of K310 acetylation (Lanzillotta et al., 2010). Notably, protective ischemic preconditioning and harmful ischemia induced similar levels of p50/RelA activation, but only the ischemic injury induced the derangement of RelA acetylation.

With the goal of optimizing the RelA acetylation state and concomitantly increasing histone acetylation, we studied the association of the specific class I HDAC inhibitor entinostat (MS-275) (Simonini et al., 2006) with resveratrol (Baur and Sinclair, 2006). MS-275 is a synthetic benzamide derivative that has been shown to inhibit HDAC 1-3 with excellent pharmacokinetic properties (Simonini et al., 2006). MS-275 has demonstrated anti-tumor activity and is currently under evaluation in phase II clinical trials for cancer therapy (Tan et al., 2010). Resveratrol is a widely studied polyphenol endowed with antiaging, anti-carcinogenic, anti-inflammatory and anti-oxidant properties (reviewed in Yu et al., 2012). It is also under clinical evaluation for the treatment of cancer, metabolic disorders, cardiovascular diseases and cognitive dysfunction in old patients (Smoliga et al., 2011). Moreover, resveratrol has shown beneficial effects in various models of brain ischemia, by interacting with a range of pathways devoted to enhance cellular stress resistance (Robb and Stuart, 2010) and mitigate the formation of free radical species and mitochondria-mediated apoptosis (Agrawal et al., 2011; Li et al., 2011; Morris et al., 2011; Ren et al., 2011). Resveratrol acts as a multi-functional drug through major activation of sirtuin 1 (Howitz et al., 2003) and stimulation of AMP-activated kinase (AMPK), a serine-threonine kinase that acts as a key metabolic balance and stress sensor/effector (Ruderman et al., 2010).

Our results demonstrate that the drug combination, by shaping RelA and histone acetylation, elicits a synergistic neuroprotection and effectiveness, even when administered $7 \mathrm{~h}$ after the onset of ischemia.

\section{Methods}

\section{Cell cultures}

Primary cultures of mouse cortical neurons

C57BL/6 mice were purchased from Charles River Italia. Primary cortical neurons were prepared from cortices of 15-day embryonic mice and cultured as previously described (Sarnico et al., 2009). Cells were plated at a density of $1.0 \times 10^{5}$ cells $/ \mathrm{cm}^{2}$ in $2 \mathrm{~cm}^{2}$ culture dishes for the viability studies, in $21 \mathrm{~cm}^{2}$ culture dishes for Western blot and co-immunoprecipitation analyses and in $56 \mathrm{~cm}^{2}$ culture dishes (Nunc, Langenselbold, Germany) for chromatin immunoprecipitation (ChIP) assays. Experiments were carried out at 11 days in vitro (DIV).

\section{$O G D$}

Oxygen glucose deprivation (OGD) was performed in cortical neurons for $3 \mathrm{~h}$ as previously described (Sarnico et al., 2009). Control cell cultures were incubated in a normal aerated incubator for the same time period. At the end of the OGD period, cells were transferred to recover in Neurobasal medium containing 0.4\% B27 supplement with or without MS-275 $(0.1,0.5$ or $1 \mu \mathrm{M})$ and resveratrol $(1,3$ or $30 \mu \mathrm{M}$ ) alone or in combination. The HDAC inhibitor MS-275 (Vinci Biochem, Italy) and resveratrol (Merck Chemicals Limited, UK) were dissolved in dimethyl sulfoxide (DMSO) and diluted before application to a final DMSO concentration lower than $0.3 \%$. When present, the AMPK inhibitor, compound C (10 $\mu \mathrm{M}$, Sigma Aldrich, Missouri, USA), or the sirtuin 1 inhibitor, sirtinol (50 $\mu \mathrm{M}$, Sigma Aldrich), were co-administered with resveratrol and MS-275. The cell viability was estimated $24 \mathrm{~h}$ later. Extraction of cell proteins or chromatin immunoprecipitation (ChIP) was performed $2 \mathrm{~h}$ after the OGD period.

Neuronal injuries were evaluated by measuring the amount of lactate dehydrogenase (LDH) released into the culture medium relative to total releasable LDH, using the CytoTox $96^{\circledR}$ Non-Radioactive Cytotoxicity Assay (Promega Corporation, Wisconsin, USA).

\section{Transient middle cerebral artery occlusion model}

Transient middle cerebral artery occlusion (tMCAO) was carried out as previously described (Pignataro et al., 2008) in male C57BL/6 mice weighing 25 to $27 \mathrm{~g}$ (Charles River Italia). Anesthesia was induced with $5 \%$ isoflurane in a $70 \%$ nitrous oxide/ $30 \%$ oxygen mixture and maintained with $2 \%$ isoflurane. The right carotid bifurcation was exposed, and the external carotid artery was coagulated distal to the bifurcation. A 5-0 nylon filament was inserted through the external carotid artery stump and advanced into the right internal carotid artery until it blocked the origin of the MCA. After 60 min of MCAO, animals were again anesthetized, and the filament was withdrawn to restore blood flow.

Cerebral blood flow (CBF) was monitored in the cerebral cortex ipsilateral to the occluded MCA with a laser-Doppler flowmeter (Periflux system). Once a stable CBF signal was obtained, the MCA was occluded. CBF was monitored throughout the $1 \mathrm{~h}$ occlusion period and the first $30 \mathrm{~min}$ of reperfusion. Only those mice that reached at least $70 \%$ of $C B F$ reduction after the MCAO were included in the experimental groups (Pignataro et al., 2008).

\section{Evaluation of ischemic volume and neurologic deficit scores}

Mice were decapitated 24 or $72 \mathrm{~h}$ after ischemia. Ischemic volume was evaluated by 2,3,5-triphenyltetrazolium chloride staining (Bederson et al., 1986). The brains were cut into $500 \mu \mathrm{m}$ coronal slices with a vibratome (Campden Instrument, $752 \mathrm{M}$ ). Sections were incubated in 2\% 2,3,5-triphenyltetrazolium chloride for $20 \mathrm{~min}$ and in $10 \%$ formalin overnight. The infarcted area was calculated by image analysis software (Image-Pro Plus) (Pignataro et al., 2008). The total infarct volume was expressed as a percentage of the volume of the hemisphere ipsilateral to the lesion.

In mice, 24 or $72 \mathrm{~h}$ after ischemia, neurological function was scored according to two scales: a general neurological scale and a focal neurological scale, as described by Clark et al. (1997). In the general score, 6 general deficits were measured: (a) hair conditions ( $0-2)$, (b) position of ears $(0-2)$, (c) eye conditions ( $0-4),(d)$ posture $(0-4)$, (e) spontaneous activity (0-4), and (f) epileptic behavior (0-12). For each of the six general parameters measured, animals received a score that correlated directly with 
the degree of symptom severity, as previously reported (Clark et al., 1997). The scores of investigated items were then summed to provide a total general score ranging from 0 to 28. For the focal score, 7 areas were assessed: (a) body symmetry, (b) gait, (c) climbing, (d) circling behavior, (e) front limb symmetry, (f) compulsory circling, and (g) whisker response. The severity of each of these items was rated on a scale from 0 to 4 . The seven items were then summed to give a total focal score ranging between 0 and 28. The single item does not provide any significant information per se. A higher score correlated with the worst animal condition (Clark et al., 1997).

Ischemic volume, neurologic function, and animal survival were evaluated in a blinded manner.

\section{Experimental protocol}

MS-275 and resveratrol were dissolved in saline and intraperitoneally (ip) administered at $1,3,5$ or $7 \mathrm{~h}$ after stroke onset. The doses used were 68,680 and $6800 \mu \mathrm{g} / \mathrm{kg}$ for resveratrol and 2, 20 and $200 \mu \mathrm{g} / \mathrm{kg}$ for MS-275. Control mice undergoing MCAO received saline in the same volume and on the same time schedule as the MS-275 or resveratrol-treated animals.

The doses used in vivo were chosen in the attempt to obtain in the cerebrospinal fluid (CSF, $50 \mu \mathrm{l}$ ) pick concentrations similar to those effective in vitro. By considering that only $2 \%$ of plasmatic resveratrol can cross the blood brain barrier (BBB) (Asensi et al., 2002), to obtain a resveratrol concentration of $3 \mu \mathrm{M}$ in the CSF, we injected a dose of $68 \mu \mathrm{g} / \mathrm{kg}$ as starting dose. Also, we approximated a total absorption through the ip route of administration and a compensation of drug metabolism by increased BBB permeability after brain ischemia.

Dosages of MS-275 were chosen in order to predict an initial CSF concentration 30 fold lower than that of resveratrol.

We also administered the drugs at 10- and 100-fold higher doses. Mice were sacrificed 24 or $72 \mathrm{~h}$ after the MCAO. Body temperature, $\mathrm{PaO}_{2}, \mathrm{PaCO}_{2}$ and $\mathrm{pH}$ values were monitored for the entire experiment both in control and drug-treated groups. Animals were randomly assigned either to the saline or the compound treatment groups.

\section{Co-immunoprecipitation and Western blot analysis}

Co-immunoprecipitation and Western blot studies of RelA and histone acetylation were performed in nuclear extracts (Pizzi et al., 2005) from mouse cortical neurons exposed to 3 h-OGD and then reoxygenated for $2 \mathrm{~h}$ in the presence of MS-275 and resveratrol or vehicle.

Twenty micrograms of nuclear extracts were diluted in RIPA buffer (10 mM Tris- $\mathrm{HCl}$ pH 8, $140 \mathrm{mM} \mathrm{NaCl}, 0.5 \%$ (v/v) Nonidet P-40, $1 \mathrm{mM}$ sodium orthovanadate, $0.1 \%$ SDS, $1 \mathrm{mM}$ phenylmethylsulfonyl fluoride, $1 \%$ protease inhibitor cocktail) and incubated at $4{ }^{\circ} \mathrm{C}$ overnight with $2 \mu \mathrm{g} / \mathrm{ml}$ of goat anti-RelA antibody (sc-372G, Santa Cruz Biotechnology, California, USA).

After incubation, $25 \mu \mathrm{l}$ of protein A/G Plus-Agarose (sc-2003, Santa Cruz Biotechnology) were added to the reaction mixture and rotated for $2 \mathrm{~h}$ at $4{ }^{\circ} \mathrm{C}$. Immunoprecipitates were collected, by centrifuging at $770 \mathrm{~g}$ for $5 \mathrm{~min}$, and washed with the RIPA buffer, as recommended by the protein A/G Plus-Agarose manufacturer. The procedure was repeated four times. After the final wash, all proteins that adhered to the protein $A / G$ beads were detached by boiling the beads in the loading buffer. Samples were rapidly centrifuged to pellet the agarose beads, and supernatants were analyzed by SDS-polyacrylamide gel electrophoresis. Co-immunoprecipitated proteins were detected by Western blotting using the following antibodies: rabbit anti-RelA (1:1000, sc-372, Santa Cruz Biotechnology), rabbit anti-acetyl-NF-кB RelA (K310) (1:250, \#3045, Cell Signaling Technology, Massachusetts, USA) and rabbit anti-acetyl-K (1:500 \#06-933 Upstate-Millipore, Massachusetts, USA).

For Western blot analyses, nuclear or cytoplasmic proteins (25 $\mu \mathrm{g}$ proteins/sample) were resolved by $4-12 \%$ SDS-polyacrylamide gels. Immunodetection was performed by incubating the membrane overnight at $4{ }^{\circ} \mathrm{C}$, with the following primary antibodies: rabbit polyclonal antiacetyl-H3 (K9/18) (1:500, \#07-593 Upstate-Millipore), rabbit polyclonal anti-histone H3 (1:1000, \#9715 Cell Signaling Technology), rabbit polyclonal anti-phospho-AMPK $\alpha$ (Thr172) (1:800, \#2535 Cell Signaling Technology) and rabbit polyclonal anti-AMPK $\alpha 1 / 2$ (1:1000, sc-25792, Santa Cruz Biotechnology).

The immunoreaction was visualized by a $1 \mathrm{~h}$ incubation at $37{ }^{\circ} \mathrm{C}$ with secondary antibodies coupled to horseradish peroxidase (1:5000, NA934, GE Healthcare, England, UK) and chemoluminescence detection using ECL Western blotting reagents (RPN2132, GE Healthcare).

Quantification of protein expression was performed by the densitometry analysis of the immunoblots using Gel Pro.3 analysis software (MediaCybernetics, MD, USA).

\section{HDAC analysis}

Total HDAC activity was measured using the EpiQuik HDAC Activity/Inhibition Assay Kit (Epigentek, New York, USA). In brief, the nuclear extracts of mouse cortical neurons, exposed to $3 \mathrm{~h}$ of OGD and subsequently treated with MS-275 and resveratrol for $2 \mathrm{~h}$, were incubated with a specific substrate for $1 \mathrm{~h}$ at $37^{\circ} \mathrm{C}$, followed by incubation with capture antibodies for $60 \mathrm{~min}$ and detection antibodies for $30 \mathrm{~min}$ at room temperature. Absorbance was determined using a microplate spectrophotometer at $450 \mathrm{~nm}$. HDAC activity was measured according to the manufacturer's instructions. All experiments were performed in triplicate at least three times before calculating the means and standard errors.

\section{Reporter gene assays}

For reporter gene fusion experiments we used: 1 ) the $B c l-x_{L}$ promoter luciferase and the $B c l-x_{L} \Delta \kappa B$ luciferase, carrying a mutation of the $\kappa B$ site, produced by Ron Hay (University of Dundee, UK) and kindly provided by Dr. Perkins (University of Dundee, UK) (Rocha et al., 2003) and 2) the Bim promoter luciferase and the Bim $\triangle \kappa B$ luciferase, carrying a mutation in the $\kappa \mathrm{B}$ site (Inta et al., 2006). After 10 DIV cortical neurons were transfected using Lipofectamine 2000 (Life Technologies, California, USA) with $1 \mu \mathrm{g} / \mathrm{ml}$ of the Bim or Bcl- $x_{L}$ expression plasmids, as previously described (Lanzillotta et al., 2010; Sarnico et al., 2009).

To investigate Bim promoter activity after drug treatments, cortical neurons were transfected with $1 \mu \mathrm{g} / \mathrm{ml}$ of the Bim expression plasmid. After $24 \mathrm{~h}$, neurons were exposed to $3 \mathrm{~h}$ OGD and $4 \mathrm{~h}$ recovery in Neurobasal medium containing 0.4\% B27 supplement with MS-275 $0.1 \mu \mathrm{M}$ and resveratrol $3 \mu \mathrm{M}$, alone or in combination.

To normalize the transfection efficiency, $0.05 \mu \mathrm{g}$ per well of a Renilla luciferase (phRLTK) control plasmid (Promega Corporation) was used, and firefly and Renilla luciferase activity were measured using a Dual Luciferase Reporter Assay (Promega Corporation). All experiments were performed in triplicate at least three times before calculating the means and standard errors.

\section{Chromatin immunoprecipitation assay and real-time PCR analysis}

Chromatin immunoprecipitation (ChIP) assays were performed to study RelA interactions and $\mathrm{H} 3$ histone acetylation at the Bim and $B c l-x_{L}$ promoters in both neuronal cultures exposed to OGD and cortices of mice subjected to MCAO. We used a ChIP assay kit (\#9003S, Cell Signaling Technology) for both cell cultures and brain tissues. Primary cultures of mouse cortical neurons, exposed to $3 \mathrm{~h}$ of OGD and then treated with MS-275 at $0.1 \mu \mathrm{M}$ and resveratrol at $3 \mu \mathrm{M}$ for $2 \mathrm{~h}$, were cross-linked with $1 \%$ formaldehyde for $10 \mathrm{~min}$ at $37^{\circ} \mathrm{C}$.

For in vivo experiments, mice were exposed to MCAO, treated with the combination of MS-275 at $2 \mu \mathrm{g} / \mathrm{kg}$ and resveratrol at $68 \mu \mathrm{g} / \mathrm{kg}$ at the beginning of reperfusion and sacrificed $3 \mathrm{~h}$ later. Frozen brain tissue from the ipsilateral hemispheres of 3 animals per group were pulled to obtain approximately $500 \mathrm{mg}$ and chopped into small pieces. Minced tissue was cross-linked with $1.5 \%$ formaldehyde for $10 \mathrm{~min}$ at $37^{\circ} \mathrm{C}$. 
For both chromatin preparations, a glycine solution was added for $5 \mathrm{~min}$ at room temperature to stop the reaction. Cells and tissues were washed with ice-cold PBS and incubated on ice for $10 \mathrm{~min}$ in a lysis buffer supplemented with $100 \mathrm{mM}$ phenylmethylsulfonyl fluoride (PMSF), dithiothreitol (DTT), and a protease cocktail inhibitor mix. Nuclei were pelleted and resuspended in a buffer supplemented with DTT, digested by micrococcal nuclease, and homogenized on ice. After centrifugation, the sheared chromatin was incubated with anti-acetyl H3 (K9/18) (\#07-593, Upstate-Millipore), anti-RelA (sc-372X, Santa Cruz Biotechnology) or anti-IgG (negative control) overnight at $4{ }^{\circ} \mathrm{C}$. Then, magnetic-coupled protein $\mathrm{G}$ beads were added and the chromatin was incubated for $2 \mathrm{~h}$ in rotation. An aliquot of chromatin that was not incubated with an antibody was used as the input control sample. Antibody-bound protein/DNA complexes were washed, eluted, treated with proteinase digest proteins, and subjected to real-time polymerase chain reaction (qRT-PCR) analyses.

Immunoprecipitated DNA ( $4 \mu \mathrm{l})$ was amplified in a $25 \mu \mathrm{l}$ reaction mixture containing SYBR Green master mix (BIORAD, California, USA). The primers used to amplify the mouse Bim promoter were as follows: forward, 5-CTG GAT GCA GGT TGG GTA G-3; and reverse, 5-GGG AAT GAG AAA GTT AGC TGG A-3, which generated a 410-bp product. The primers used to amplify the $B c l-x_{L}$ promoter were as follows: forward, 5-CTC AGC TCT CCA GCA CAC AC-3; and reverse, 5-ACA ACA TGG CCT CAG GAA AA-3, which generated a 160-bp product. Incorporation of the SYBR Green dye into the PCR products was monitored in real-time with a BIORAD iCycler detection system, allowing the determination of the threshold cycle $\left(C_{T}\right)$ at which the exponential amplification of PCR products began. Each reaction was performed in triplicate.

In ChIP experiments, $C_{\mathrm{T}}$ values obtained by qRT-PCR analysis in samples immunoprecipitated with anti-RelA or anti-acetyl-H3 antibody were normalized over corresponding $C_{T}$ values obtained by IgGimmunoprecipitation, and further normalized over relative $C_{T}$ values obtained in INPUT (no antibody) chromatin. Final data obtained in neurons exposed to OGD or OGD plus drugs were then normalized to data obtained in control neurons, i.e., neurons maintained in normal oxygen-glucose conditions. Data obtained in mice subjected to MCAO or MCAO plus drugs were normalized to the data obtained in shamoperated mice. Bars represent the fold changes relative to control cells in Figs. 1D and 4D, or to sham-operated mice in Fig. 7.

\section{Statistics}

Data obtained in cultured neurons were expressed as mean \pm standard error and statistical significance of differences between groups was evaluated by two-way ANOVA and Dunnet's multiple comparison test, using GraphPad Prism 5 software (GraphPad Software, Inc.). $p<0.05$ was considered to be significant. Data from ChIP analyses were analyzed using Student's $t$-tests for independent data. $p<0.05$ was considered to be significant.

For the evaluation of the protective effect of MS-275 and resveratrol in vivo, on the infarct volume, data were expressed as mean \pm standard error and statistical analysis was performed by 2-way ANOVA followed by Newman Keuls test.

Data relative to focal and general neurological deficits, being ordinal data, were analyzed using the non-parametric Kruskal-Wallis test, followed by the Nemenyi test for the non-parametric multiple comparison. Statistical significance was accepted at the 95\% confidence level $(p<0.05)$.

\section{Study approval}

All animal experiments were authorized by the Italian Ministry of Health and by the Animal Care and Use Committees at the University of Brescia and the University of Naples "Federico II" in compliance with the Italian guidelines for animal care and use (DL 116/92) and the European Communities Council Directive (86/609/EEC).

\section{Results}

Histone H3 and RelA acetylation levels in neurons following OGD

We investigated whether the histone $\mathrm{H} 3$ acetylation state was altered in primary cultures of mouse cortical neurons exposed to $3 \mathrm{~h}$ of OGD. This is an experimental condition leading to neuronal apoptosis within a few hours and secondary plasma membrane breakdown (Sarnico et al., 2009), evaluated through the measurement of LDH released in the medium at $24 \mathrm{~h}$. Western blot analysis of nuclear extracts prepared after $2 \mathrm{~h}$ of reoxygenation revealed a significant decrease in the ratio between the histone $\mathrm{H} 3$ acetylation at the Lys9/18 (H3 Ac-K9/18) and the total histone $\mathrm{H} 3(p=0.0421, \mathrm{~F}=3.16$, Fig. $1 \mathrm{~A})$. We also evaluated the acetylation state of the NF-kB RelA. RelA was immunoprecipitated from nuclear extracts, and its acetylation was assessed using a specific anti-acetyl-RelA(K310) antibody (RelA Ac-K310) and the general anti-acetyl-K antibody that recognizes general lysine acetylation. As previously described, co-immunoprecipitation analysis revealed a global RelA increase after OGD ( $p=0.0054, \mathrm{~F}=19.73$ ) (Lanzillotta et al., 2010; Sarnico et al., 2009). In this condition acetylation at K310 of RelA appeared increased $(p=0.0463, \mathrm{~F}=7.01)$ despite a reduced level of overall RelA acetylation ( $p=0.0072, \mathrm{~F}=26.42$ ) (Fig. 1B) (Lanzillotta et al., 2010).

\section{OGD-promoted RelA activation induces Bim and inhibits Bcl- $x_{L}$ promoters}

We have previously shown that the exposure of cortical neurons to OGD significantly induces the expression of the pro-apoptotic Bim (Inta et al., 2006) while reducing the expression of anti-apoptotic $B c l-x_{L}$ gene (Sarnico et al., 2009). We here studied the specific contribution of NF-кB to the anoxia-induced pro-apoptotic program by investigating the responsivity of the $B i m$ and $B C l-x_{L}$ promoters to OGD.

Primary cortical neurons were transfected with Bim or $B c l-x_{L}$ promoter-luciferase plasmids or with Bim and $B c l-x_{L} \Delta \kappa B$ carrying a mutation in the sequence of the NF- $\kappa B$ binding site. As previously reported (Sarnico et al., 2009), the OGD exposure induced a twofold increase in the activity of the $\operatorname{Bim}$ promoter $(p=0.0287, \mathrm{~F}=12.33)$. No modification was induced in the Bim $\Delta \kappa B$ plasmid activity, demonstrating that changes in the Bim transcription were NF-kB-specific $(p=0.93, \mathrm{~F}=0.42)$. Conversely, the $B c l-x_{L}$ promoter activity was partially reduced by OGD exposure $(p=0.0476, \mathrm{~F}=16.21)$. The mutation of the NF- $\kappa \mathrm{B}$ binding site at the $B c l-x_{L}$ promoter reduced the basal activity and the responsiveness to $\mathrm{OGD}(p=0.0490, \mathrm{~F}=8.47)$ (Fig. 1C).

We further analyzed the interactions between RelA and the Bim or $B c l-x_{L}$ promoters induced by OGD, through chromatin immunoprecipitation (ChIP) assays. The recruitment of RelA to Bim and $B c l-x_{L}$ promoters was determined by quantitative real-time polymerase chain reaction (qRT-PCR) analyses using primers that amplified the sequence of the Bim and $B c l-x_{L}$ promoters, including the $\kappa B$ binding sites.

RelA binding to the Bim promoter increased while binding to $B c l-x_{L}$ promoter decreased. These findings suggest that, as a possible consequence of OGD-induced derangement of RelA acetylation, RelA shifts from the $B c l-x_{L}$ to the Bim promoter (Fig. 1D).

Synergistic neuroprotection elicited by MS-275 and resveratrol in cortical neurons exposed to $O G D$

In order to evaluate the neuroprotective activity of MS-275 and resveratrol, primary cortical neurons were exposed to OGD for $3 \mathrm{~h}$, and then treated with resveratrol at 1,3 or $30 \mu \mathrm{M}$ concentrations or with MS-275 at concentrations of $0.1,0.5$ or $1 \mu \mathrm{M}$. A previous evaluation of drug toxicity showed no adverse effect on cell viability (Supplementary Fig. 1A). In the OGD experimental setting, both compounds displayed per se a significant neuroprotective activity at the higher concentrations, $30 \mu \mathrm{M}$ for resveratrol $(p=0.0428, \mathrm{~F}=15.24)$ and $0.5-1 \mu \mathrm{M}$ for MS-275 ( $p=0.0437, p=0.0392$, respectively; $\mathrm{F}=6.12, \mathrm{~F}=8.15$, respectively). 
A
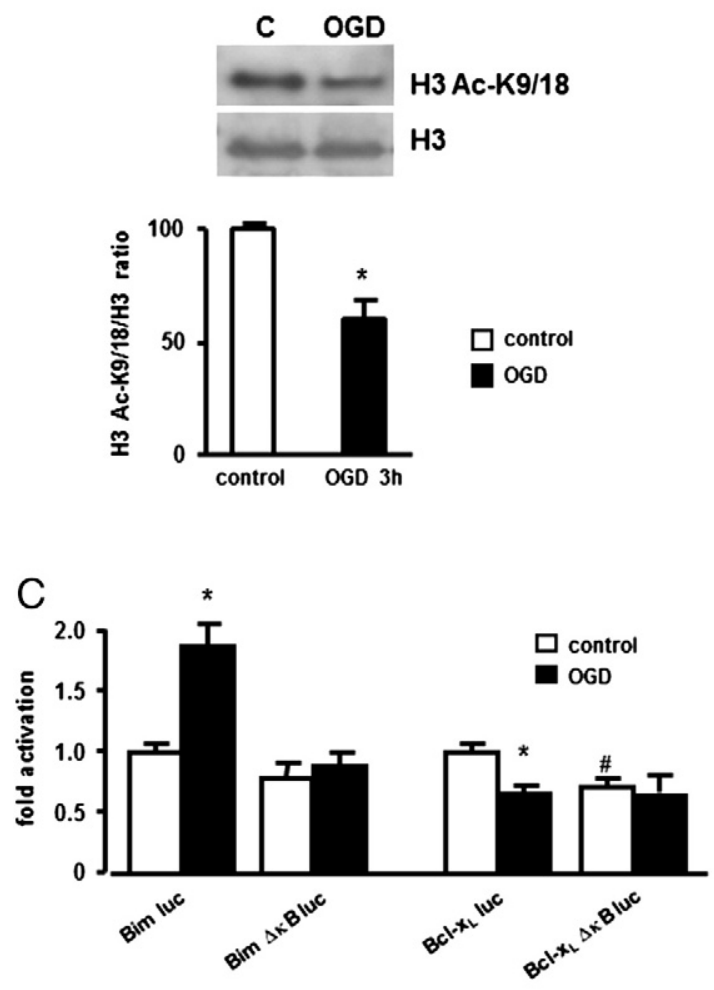

B

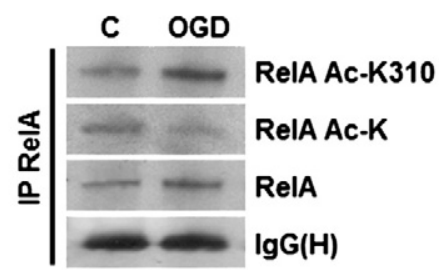

D
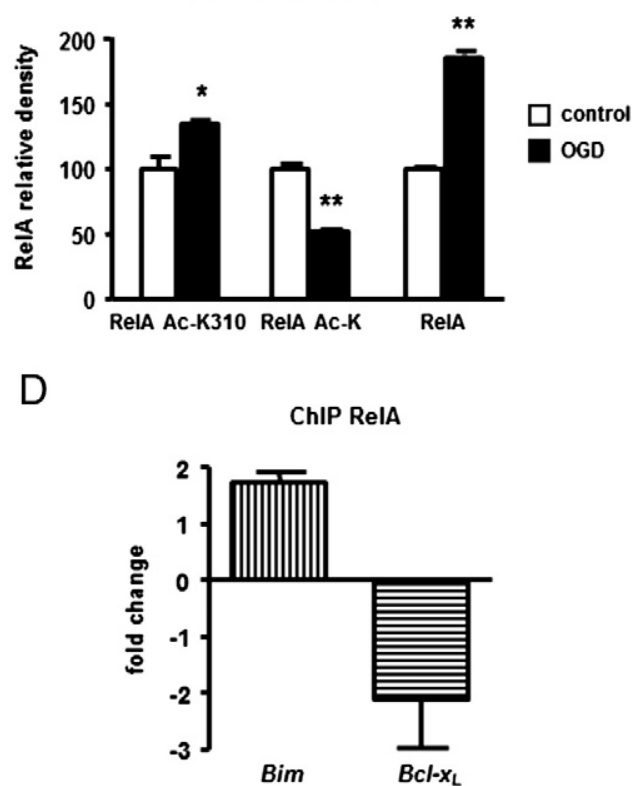

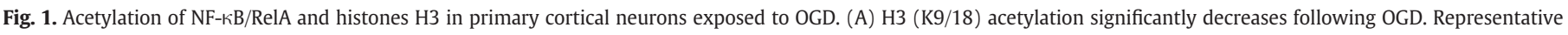

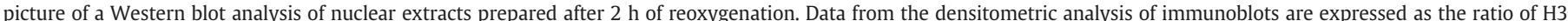

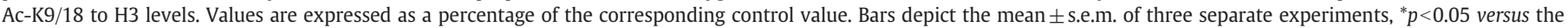

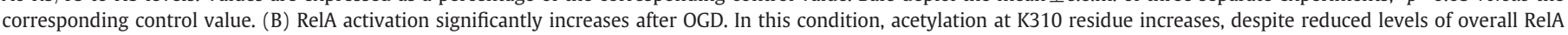

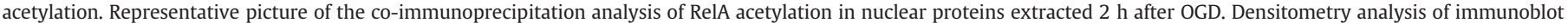

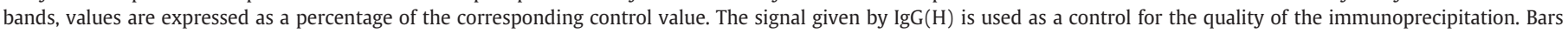

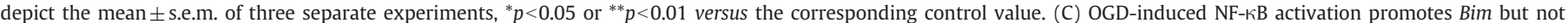

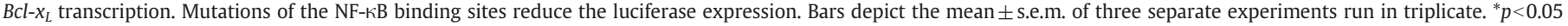

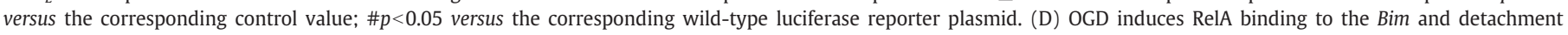

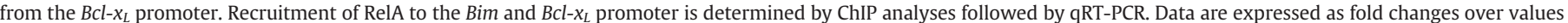
obtained in cells maintained in normal oxygen-glucose condition. All experiments $(\mathrm{N}=3)$ are performed in triplicate, bars depict the mean \pm s.e.m.

No effect was observed at the lower concentrations of $3 \mu \mathrm{M}$ for resveratrol $(p=0.93, \mathrm{~F}=2.04)$ and $0.1 \mu \mathrm{M}$ for MS-275 $(p=0.81, \mathrm{~F}=1.59)$. However, when tested in association the subthreshold concentrations of resveratrol and MS-275 maximally prevented the cell death ( $p=$ $0.0383, \mathrm{~F}=17.42$ ), suggesting that the two compounds can elicit a synergistic effect (Fig. 2).

\section{Effect of MS-275 and resveratrol on histone $\mathrm{H3}$ acetylation}

To investigate the molecular mechanisms associated with synergistic neuroprotection elicited by resveratrol and MS-275, we investigated the capability of the two compounds to inhibit the HDAC activity and restore the normal histone acetylation. A colorimetric HDAC activity assay was used to evaluate the inhibition of class I and II HDACs in mouse cortical neurons exposed to $3 \mathrm{~h}$ of OGD and then treated with MS-275 and resveratrol during the subsequent $2 \mathrm{~h}$ of reoxygenation. The general HDAC activity was not significantly modified by OGD exposure $(p=0.35, \mathrm{~F}=1.78)$. When applied in the post-OGD period, MS-275 and resveratrol were able to reduce HDAC function. The HDAC activity showed a trend to decrease in the presence of $0.1 \mu \mathrm{M}$ MS-275 $(p=0.64, \mathrm{~F}=1.16)$ and was markedly depressed by $1 \mu \mathrm{M}$ MS-275 ( $p=0.0067,3.71)$, confirming the HDAC inhibitory activity of MS-275. A similar degree of HDAC inhibition was produced by either 3 or $30 \mu \mathrm{M}$ of resveratrol $(p=0.0042, p=$ 0.0199 , respectively; $F=4.23, F=11.98$, respectively), in agreement with the capability of resveratrol to inhibit the HDAC6 isoform
(Blackwell et al., 2008). Drugs used in combination at the lower concentrations showed an additive inhibitory effect on the general HDAC activity $(p=0.0037, F=3.69)$, suggesting that the drugs inhibit different HDAC isoforms (Blackwell et al., 2008) (Fig. 3A).

We then evaluated the pharmacological activity of MS-275 and resveratrol in modulating the acetylation state of histone $\mathrm{H} 3$ in primary cortical neurons exposed to OGD. Western blot analysis of nuclear extracts showed that MS-275 at the neuroprotective $1 \mu \mathrm{M}$ concentration $(p=0.018, \mathrm{~F}=10.66)$, but not at the $0.1 \mu \mathrm{M}$ concentration $(p=0.08, \mathrm{~F}=2.17)$ used in the synergistic combination, was able to counteract $\mathrm{H} 3$ deacetylation on K9/18. No significant effect of resveratrol on $\mathrm{H} 3$ acetylation was detected at both tested concentrations $3 \mu \mathrm{M}(p=0.77, \mathrm{~F}=1.50)$ or $30 \mu \mathrm{M}(p=0.11, \mathrm{~F}=1.44)$. Combining the two drugs at the lower concentrations completely restored the H3 acetylation ( $p=0.042, \mathrm{~F}=28.69)$, suggesting that the synergistic neuroprotection was also associated with the capability of the drugs to maintain adequate levels of histone $\mathrm{H} 3$ acetylation (Fig. 3B).

The increase in $\mathrm{H} 3$ acetylation in a cell maintained in normal oxygenglucose condition was evident by using MS-275 at the higher concentration, but not using a combination of MS-275 and resveratrol at low concentrations (Supplementary Fig. 1B).

\section{Resveratrol increases AMPK phosphorylation in the post-OGD period}

We evaluated the capability of the two compounds to increase the activity of AMP-activated kinase (AMPK) by measuring AMPK 
phosphorylation at the Thr172 (T172) residue, a known molecular target of resveratrol (Ruderman et al., 2010). Immunoblot analysis of cytoplasmic extracts showed that resveratrol at $30 \mu \mathrm{M}$ is able to increase AMPK phosphorylation, in basal conditions $(p=0.0459$ and $\mathrm{F}=24.92$, Supplementary Fig. 1C). Furthermore, AMPK dephosphorylation produced by OGD $(p<0.0001, \mathrm{~F}=21.02)$ was efficiently counteracted by resveratrol at either $3 \mu \mathrm{M}$ or $30 \mu \mathrm{M}$ concentration ( $p=0.0315, p=0.0044$, respectively; $\mathrm{F}=10.93, \mathrm{~F}=12.82$, respectively). As shown in Fig. 3C, MS-275 neither increased the AMPK phosphorylation (at $0.1 \mu \mathrm{M} p=0.90$ and $\mathrm{F}=1.87$, at $1 \mu \mathrm{M}$ $p=0.13$ and $\mathrm{F}=1.02$ ), nor reinforced the stimulatory effect produced by resveratrol at $3 \mu \mathrm{M}(p=0.0330$ and $\mathrm{F}=57.46)$. It suggests that while resveratrol at the lower concentration is able to activate the AMPK function, no synergy with MS-275 occurs at the level of AMPK ( $p=0.26$ and $\mathrm{F}=2.25$ resveratrol $3 \mu \mathrm{M}$ versus MS-275resveratrol combination).

The combination of MS-275 and resveratrol prevents the derangement of RelA acetylation and reduces both histone $\mathrm{H} 3$ acetylation and RelA recruitment at the Bim promoter

We evaluated the capability of MS-275 and resveratrol alone and in combination to revert the derangement of RelA acetylation, i.e., the general deacetylation of RelA and the specific acetylation of K310 residue, following OGD.

Treatment in the post-OGD period with MS-275 at $0.1 \mu \mathrm{M}$ or $1 \mu \mathrm{M}$ per se did not reduce the acetylation of RelA at the K310 residue ( $p=$ $0.41, p=0.39$, respectively; $\mathrm{F}=1.65, \mathrm{~F}=2.01$, respectively), but significantly increased the global RelA acetylation $(p=0.0456, p=0.0020$, respectively; $\mathrm{F}=6.99, \mathrm{~F}=12.84$, respectively). Conversely, resveratrol either at $3 \mu \mathrm{M}$ or $30 \mu \mathrm{M}$ did not increase the total RelA acetylation ( $p=0.34, p=0.06$, respectively; $\mathrm{F}=2.48, \mathrm{~F}=1.12$, respectively) but, in line with previous data (Lanzillotta et al., 2010), it reduced the K310 acetylation $(p=0.0015$ and $\mathrm{F}=19.89)$ at the higher concentration. When used together, $0.1 \mu \mathrm{M}$ MS-275 and $3 \mu \mathrm{M}$ resveratrol efficiently reverted the unbalanced RelA acetylation produced by OGD by increasing the general RelA acetylation $(p=0.0073$ and $\mathrm{F}=16.95)$ and by specifically deacetylating the $\mathrm{K} 310$ residue $(p<0.0001$ and $\mathrm{F}=$ 59.77) (Fig. 4A).
Our previous findings showed that OGD-induced Bim promoter and cell death can be significantly enhanced by RelA overexpression. Bim promoter activation and neurotoxicity dramatically decreased in cells overexpressing the acetylation resistant RelA-K310R mutant (Lanzillotta et al., 2010), indicating that NF-кB-mediated events are completely dependent on K310 acetylation of RelA. To investigate the response of the Bim promoter to the drug administration after OGD exposure, we performed experiments using reporter fusion genes. Mouse cortical neurons transfected with Bim promoter luciferase plasmid were treated with MS-275 $(0.1 \mu \mathrm{M})$ and resveratrol $(3 \mu \mathrm{M})$ individually or in combination. The OGD-induced activation of the Bim promoter was not modified by MS-275 $(p=0.82$ and $\mathrm{F}=$ $1.55)$ and was only slightly decreased by resveratrol alone $(p=0.24$ and $\mathrm{F}=2.13$ ), while it was significantly suppressed by the drug combination $(p=0.0007$ and $\mathrm{F}=10.71)$ (Fig. 4B).

We also performed ChIP assays to evaluate the acetylation of $\mathrm{H} 3$ histone and RelA binding at the Bim promoter. When compared to vehicle treatment, the drug exposure after OGD led to a strong decrease in the RelA binding and endogenous $\mathrm{H} 3$ acetylation at the promoter $(p=0.0404, p=0.0020$, respectively; $\mathrm{F}=4.55, \mathrm{~F}=30.97$, respectively) (Fig. 4C). These findings suggest that the synergistic effect elicited by MS-275 and resveratrol is preceded by a modification of RelA acetylation that causes the detachment of the NF- $\kappa B$ transcription factor from the Bim promoter and inhibits the pro-apoptotic Bim expression.

To explore a possible role of the AMPK-sirtuin 1 pathway activation in neuroprotection, we tested an MS-275 and resveratrol combination in the presence of compound C, an AMPK inhibitor, or sirtinol, a sirtuin 1 inhibitor. Both AMPK and sirtuin 1 inhibition completely blocked the neuroprotective effect of the MS-275 and resveratrol combination $(p=$ 0.46 and $\mathrm{F}=1.36$ for compound $\mathrm{C} ; \mathrm{p}=0.98$ and $\mathrm{F}=1.18$ for sirtinol) (Fig. 4D), suggesting that AMPK-sirtuin 1 pathway is deeply involved in synergistic neuroprotection.

The MS-275 and resveratrol combination reduces MCAO-induced brain damage and ameliorates focal and general scores

For the mouse ischemia model, the right middle cerebral artery was occluded for $60 \mathrm{~min}$, followed by $24 \mathrm{~h}$ of reperfusion. Treatments were administered ip at the beginning of reperfusion period. To determine the synergistic protective effect of the association between MS-275

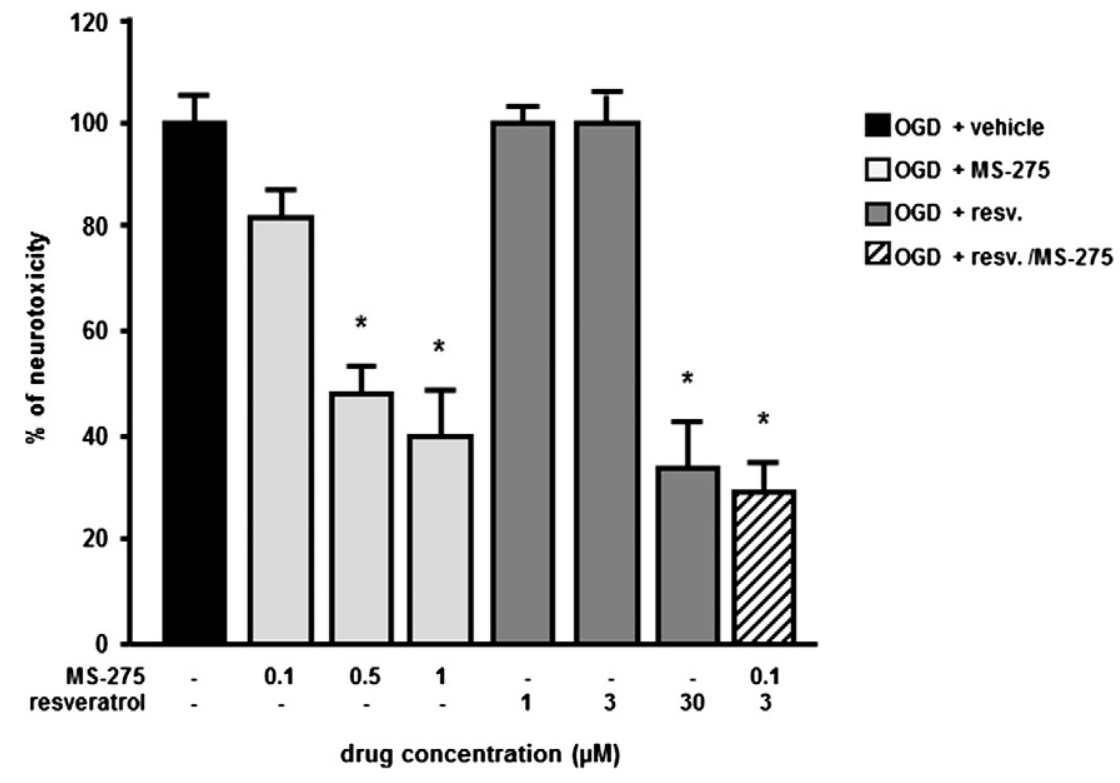

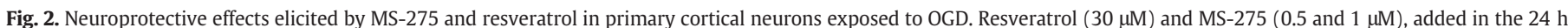

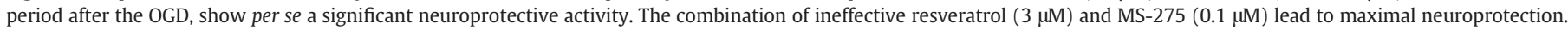

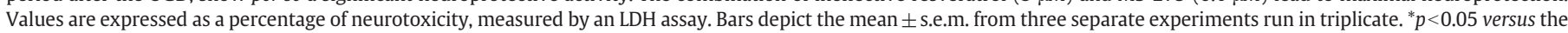
corresponding OGD value. 

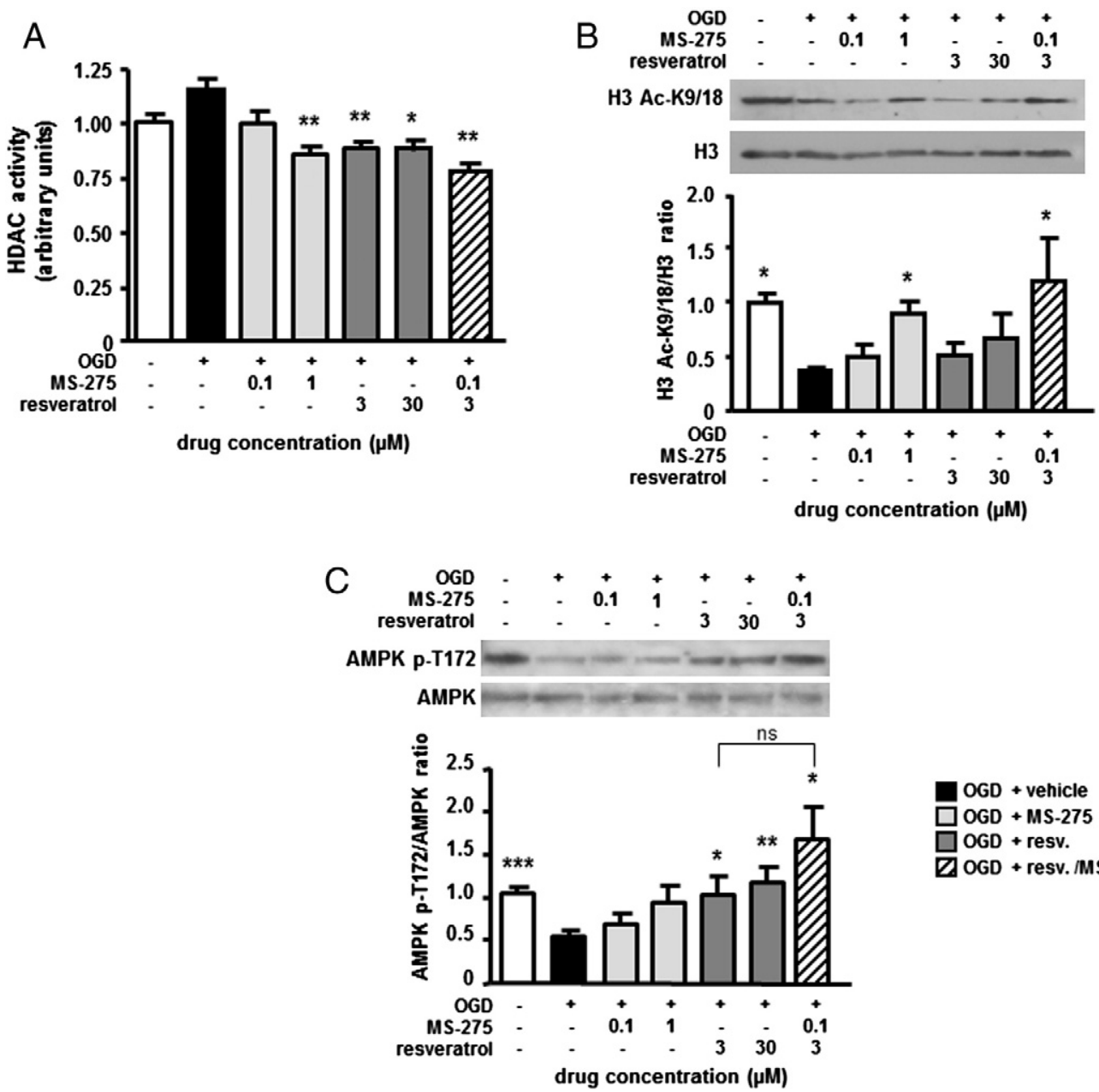

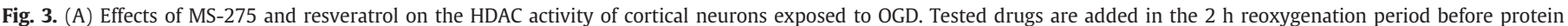

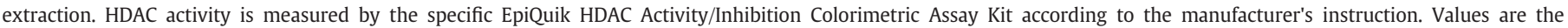

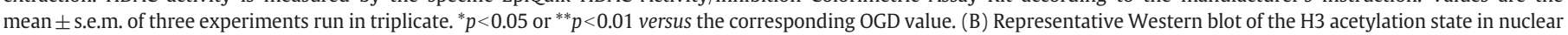

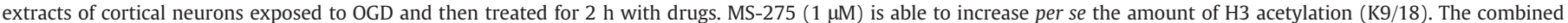

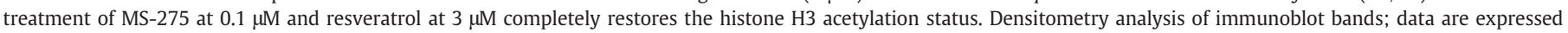

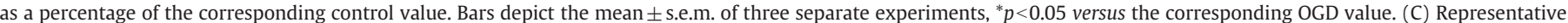

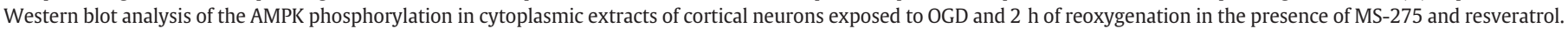

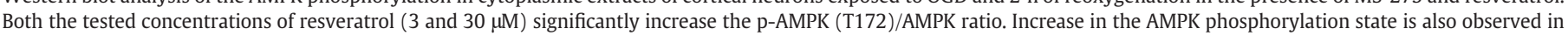

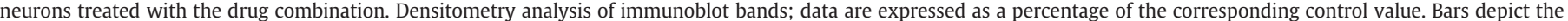
mean \pm s.e.m. of three separate experiments, ${ }^{*} p<0.05,{ }^{* *} p<0.01$ or ${ }^{* * *} p<0.001$ versus the corresponding OGD value.

and resveratrol, the two compounds were injected individually at increasing doses (resveratrol, 68, 680, 6800 $\mu \mathrm{g} / \mathrm{kg}$; MS-275, 2, 20 and $200 \mu \mathrm{g} / \mathrm{kg}$ ), or in combination (resveratrol $68 \mu \mathrm{g} / \mathrm{kg}+\mathrm{MS}-2752 \mu \mathrm{g} / \mathrm{kg}$ and resveratrol $680 \mu \mathrm{g} / \mathrm{kg}+\mathrm{MS}-27520 \mu \mathrm{g} / \mathrm{kg}$ ). Administration of $2 \mu \mathrm{g} / \mathrm{kg}$ MS-275 ( $\mathrm{n}=5$, infarct volume $=58.5 \pm 0.9 \%), 68 \mu \mathrm{g} / \mathrm{kg}$ resveratrol $(\mathrm{n}=7$, infarct volume $=58.4 \pm 4.2 \%)$ and $680 \mu \mathrm{g} / \mathrm{kg}$ resveratrol $(n=6$, infarct volume $=53.1 \pm 3.6 \%)$ alone did not induce a significant difference in the infarct volume $(p=0.54, p=0.61, p=0.16$, respectively; $\mathrm{F}=0.40, \mathrm{~F}=0.27, \mathrm{~F}=2.38$, respectively), while $6800 \mu \mathrm{g} / \mathrm{kg}$ resveratrol $(n=5$, infarct volume $=35.8 \pm 5.8 \%), 20 \mu \mathrm{g} / \mathrm{kg}$ MS-275 $(\mathrm{n}=$ 5 , infarct volume $=48.2 \pm 3.6 \%)$ and $200 \mu \mathrm{g} / \mathrm{kg}$ MS-275 $(\mathrm{n}=5$, infarct volume $=33.1 \pm 6.8 \%$ ) were able to produce a significant reduction in the infarct volume $(p=0.004, p=0.047, p=0.004$, respectively; $\mathrm{F}=$ $13.34, F=5.51, F=14.25$, respectively) compared with the vehicle treated group $(n=6$, infarct volume $=61.2 \pm 3.8 \%)$. Though, a maximal $30 \%$ protection was produced by the highest doses for both drugs.

Remarkably, a more consistent reduction in the infarct volume was observed when ischemic mice were treated with the combination of the two compounds at the lowest doses, $68 \mu \mathrm{g} / \mathrm{kg}$ resveratrol plus $2 \mu \mathrm{g} / \mathrm{kg}$ MS-275 $(\mathrm{n}=7$, infarct volume $=14.8 \pm 1.1 \%$ ). The synergistic effect did not improve when a combination of 10-fold higher dose for both drugs was used, i.e., $680 \mu \mathrm{g} / \mathrm{kg}$ resveratrol plus $20 \mu \mathrm{g} / \mathrm{kg}$ MS-275 ( $\mathrm{n}=5$, infarct volume $=27.8 \pm 5.7 \%)(p=0.0001$ and $p=0.0001$, respectively; $\mathrm{F}=158.4$ and $\mathrm{F}=24.54$, respectively) (Fig. $5 \mathrm{~A}$ ).

The general (Fig. 5B) and focal score (Fig. 5C) evaluations confirmed the protective activity of the drug combination, though some ameliorations were also detected for separate treatments. Not any of the tested treatments induced a change in neurobehavioral scores in the sham-operated animals (data not shown).

The numbers of animals excluded, because they died during the surgery procedures or because the reduction in the CBF was less than 70\%, were respectively: vehicle-treated group, 3; $68 \mu \mathrm{g} / \mathrm{kg}$ resveratrol, $1 ; 680 \mu \mathrm{g} / \mathrm{kg}$ resveratrol, $1 ; 6800 \mu \mathrm{g} / \mathrm{kg}$ resveratrol, $0 ; 2 \mu \mathrm{g} / \mathrm{kg}$ MS-275, 1; $20 \mu \mathrm{g} / \mathrm{kg}$ MS-275, 0; $200 \mu \mathrm{g} / \mathrm{kg}$ MS-275: 1; $68 \mu \mathrm{g} / \mathrm{kg}$ resveratrol $+2 \mu \mathrm{g} / \mathrm{kg}$ MS-275, 2; $680 \mu \mathrm{g} / \mathrm{kg}$ resveratrol $+20 \mu \mathrm{g} / \mathrm{kg}$ MS-275, 1. 

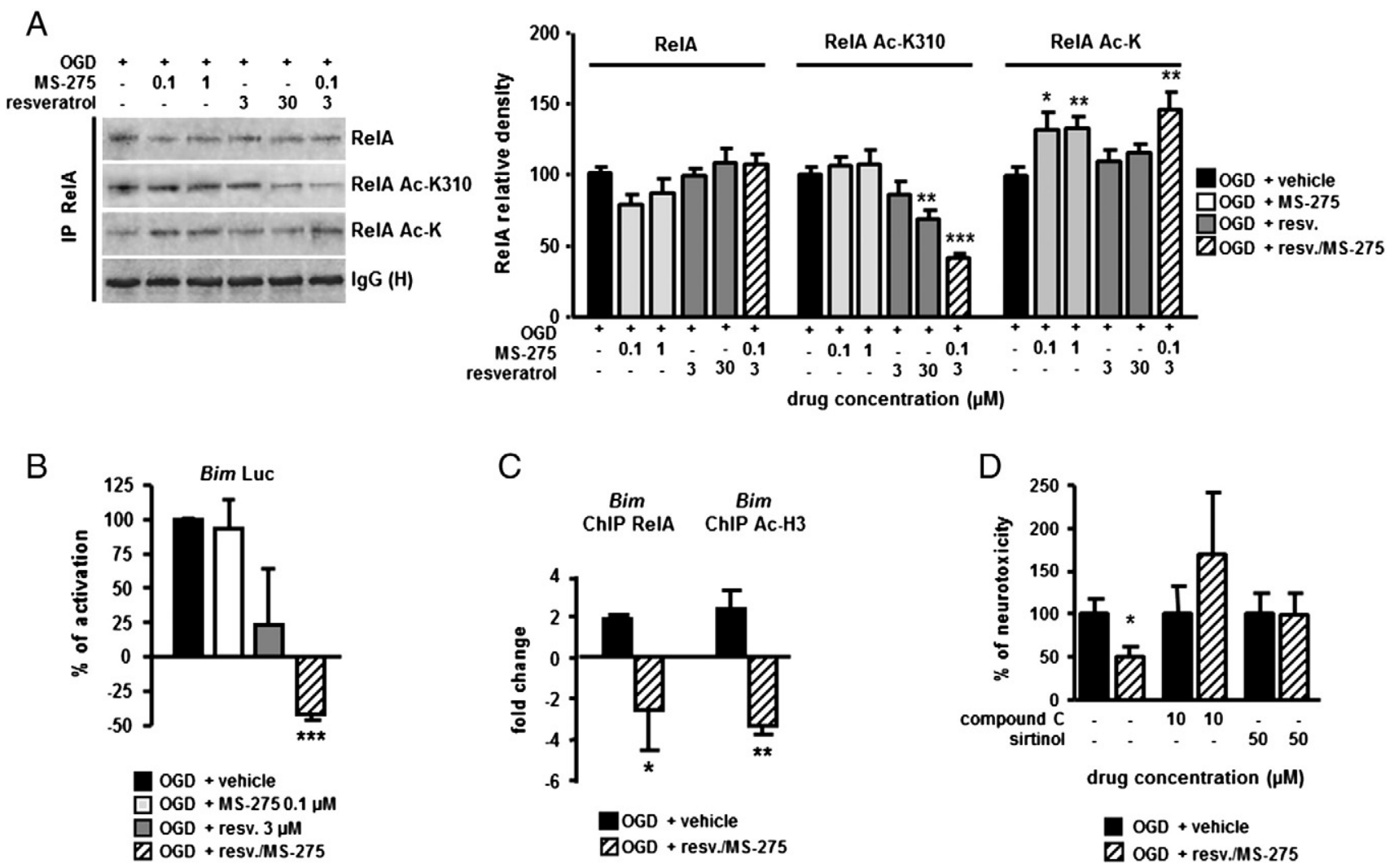

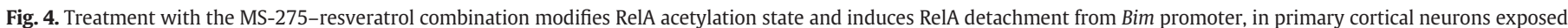

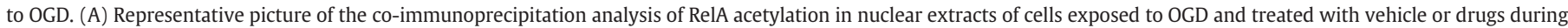

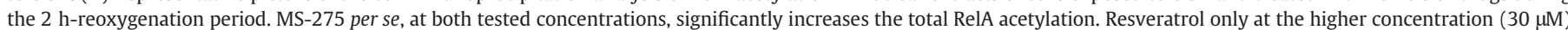

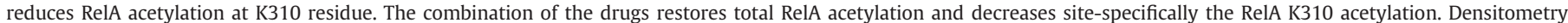

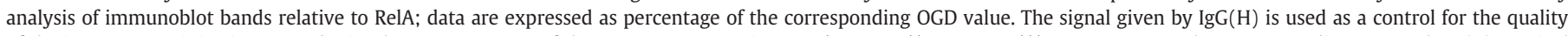

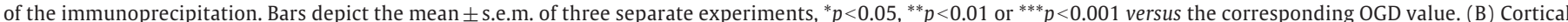

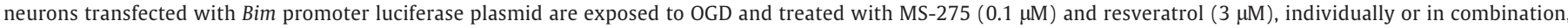

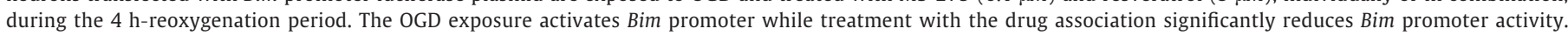

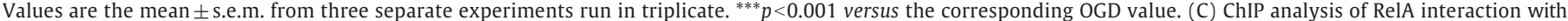

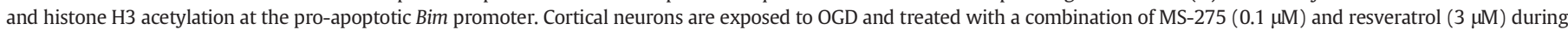

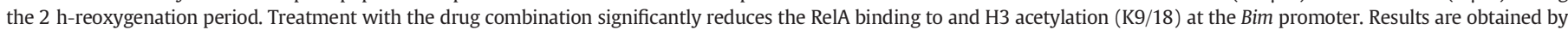

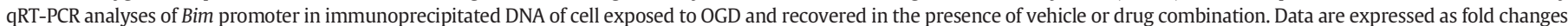

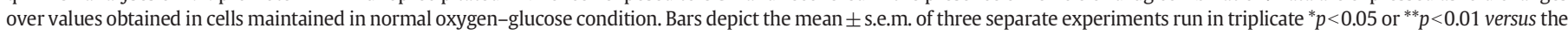

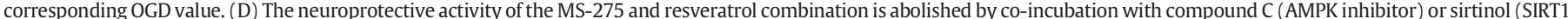

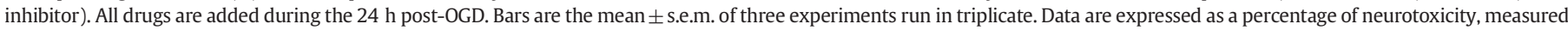
by an LDH assay. ${ }^{*} p<0.05$ versus the corresponding OGD value.

To verify whether the protection was persistent for more than $24 \mathrm{~h}$, another set of experiments was performed, and animals were sacrificed $72 \mathrm{~h}$ after stroke onset. Interestingly, the combination of the singularly ineffective dosages of resveratrol and MS-275 was able to induce a significant reduction $(p=0.0001$ and $\mathrm{F}=393.73)$ in the infarct volume $(n=7$, infarct volume $=16.2 \pm 1.2 \%)$ compared to vehicle-treated animals $(n=6$, infarct volume $=58.4 \pm 1.8 \%$ ), $2 \mu \mathrm{g} / \mathrm{kg}$ MS-275-treated animals $(n=5$, infarct volume $=46.1 \pm 4.6 \%, p=0.089$ and $\mathrm{F}=3.62)$ and $68 \mu \mathrm{g} / \mathrm{kg}$ resveratrol-treated animals $(\mathrm{n}=5$, infarct volume $=50.6 \pm 2.0 \%, p=0.19$ and $F=2.00$ ) (Supplementary Fig. 2A). These data were accompanied by an amelioration in the general (Supplementary Fig. 2B) and focal neurological scores (Supplementary Fig. 2C). The numbers of animals excluded, because they died during the surgery procedures or because the reduction in the CBF was less than $70 \%$ were as follows: vehicle-treated group, 3; resveratrol (68 $\mu \mathrm{g} / \mathrm{kg}), 1$; MS-275 $(2 \mu \mathrm{g} / \mathrm{kg}), 1$; resveratrol $(68 \mu \mathrm{g} / \mathrm{kg})+\mathrm{MS}-275$ (2 $\mu \mathrm{g} / \mathrm{kg}), 1$.

To detect a possible effect of MS-275 or resveratrol on other physiological parameters, $\mathrm{PaO}_{2}, \mathrm{PaCO}_{2}, \mathrm{pH}$ and body temperature were monitored in all animal groups. The two compounds did not affect any parameter at any tested dosage (data not shown). Furthermore, MS-275 and resveratrol did not modify cerebral blood flow (CBF), as measured for $3 \mathrm{~h}$ by laser Doppler and compared with vehicle-injected animals (data not shown).

The MS-275-resveratrol combination is effective in ameliorating the consequences of an ischemic stroke in mice when it is administered up to $7 \mathrm{~h}$ after ischemia onset

To determine the time window for the effectiveness of the combined MS-275 and resveratrol in treating brain ischemia, the two compounds were ip administered to ischemic mice at 1, 3, 5 and $7 \mathrm{~h}$ after MCAO. The percentage of the ipsilateral hemisphere infarct in the vehicle-treated animals was $61.2 \pm 3.8$. However, administration of the MS-275 and resveratrol combination $(2 \mu \mathrm{g} / \mathrm{kg}$ and $68 \mu \mathrm{g} / \mathrm{kg}$ respectively) at $1 \mathrm{~h}(\mathrm{n}=7), 3 \mathrm{~h}(\mathrm{n}=6)$ or $5 \mathrm{~h}(\mathrm{n}=7)$ after the MCAO significantly reduced the percentage of the infarct respectively to $14.7 \pm 1.1,30.0 \pm 6.8,29.6 \pm 5.9(p=0.0001, p=0.002$ and $p=$ 0.001 , respectively; $\mathrm{F}=158.4, \mathrm{~F}=16.04, \mathrm{~F}=18.72$, respectively) (Fig. 6A) and the neurological deficits (Figs. 6B and C). Treatment starting at $7 \mathrm{~h}$ still displayed a minor, but significant neuroprotection $(\mathrm{n}=5$, infarct volume $=44.8 \pm 6.4 \% ; p=0.049, \mathrm{~F}=5.20)$. 
The numbers of animals excluded, because they died during the surgery procedures or because the reduction in the $\mathrm{CBF}$ was less than $70 \%$, were the following: vehicle-treated group, 3 ; drug combination administered $3 \mathrm{~h}$ after MCAO, 2; drug combination administered $5 \mathrm{~h}$ after MCAO, 1; drug combination administered $7 \mathrm{~h}$ after MCAO, 1.

MS-275 and resveratrol administration after MCAO drives RelA recruitment and $\mathrm{H} 3$ acetylation from the Bim to the $B c l-x_{L}$ promoter

We previously demonstrated that derangement of RelA acetylation found in cortical neurons exposed to OGD is also present in cerebral cortices of mice exposed to a transient MCAO and $4 \mathrm{~h}$ of reperfusion (Lanzillotta et al., 2010). Here, we evaluated how the state of the RelA acetylation could affect the RelA recruitment and histone $\mathrm{H} 3$ acetylation at both the Bim and $B c l-x_{L}$ promoters in vivo. After 1-h MCAO, reperfused mice were intraperitoneally injected with the drug combination (MS-275 $2 \mu \mathrm{g} / \mathrm{kg}$ and resveratrol $68 \mu \mathrm{g} / \mathrm{kg}$ ) or with vehicle. Three hours later, mice were sacrificed and the ischemic cortices were processed for ChIP analysis. When compared to the vehicle condition, the templates of treated mice displayed a strong reduction of RelA binding and $\mathrm{H} 3$ acetylation at the Bim promoter ( $p=0.0140$ and $p=0.0022$ respectively) (Figs. 7A and B). Conversely both RelA binding and $\mathrm{H} 3$ acetylation increased at the $B c l-\chi_{L}$ promoter in treated mice $(p=0.0174$ and $p=0.0277$ respectively) (Figs. $7 C$ and D). These results suggest that in pathological conditions, the acetylation state of RelA drives the transcriptional activity of NF- $\kappa B$ towards pro-apoptotic genes. The binding of NF- $\kappa \mathrm{B}$ to a gene promoter affects the acetylation state of the promoter-associated histones to finalize the proper gene transcription.

\section{Discussion}

The present study demonstrates that epigenetic drugs targeting aberrant acetylation of NF- $\kappa \mathrm{B} /$ RelA and histone proteins reduce postischemic brain injury with a wide therapeutic window.

1h MCAO $+24 \mathrm{~h}$ reperfusion
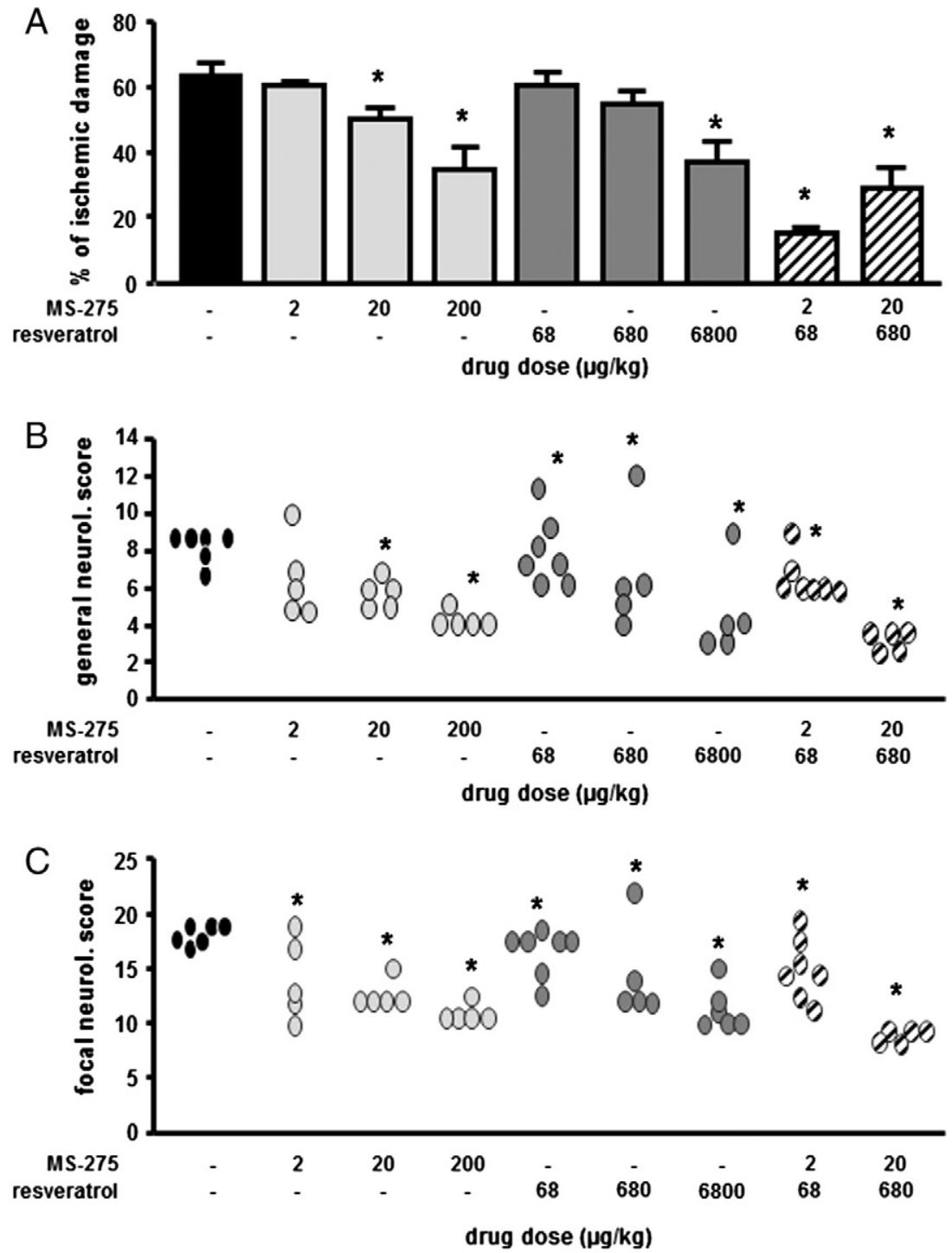

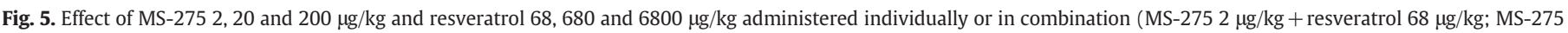

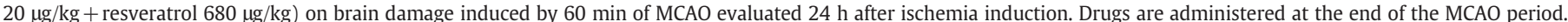

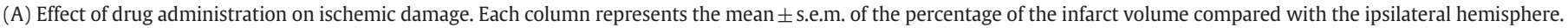

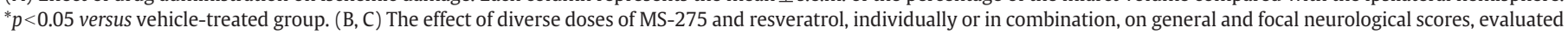
$24 \mathrm{~h}$ after the ischemia induction. ${ }^{*} p<0.05$ versus vehicle-treated animals. 

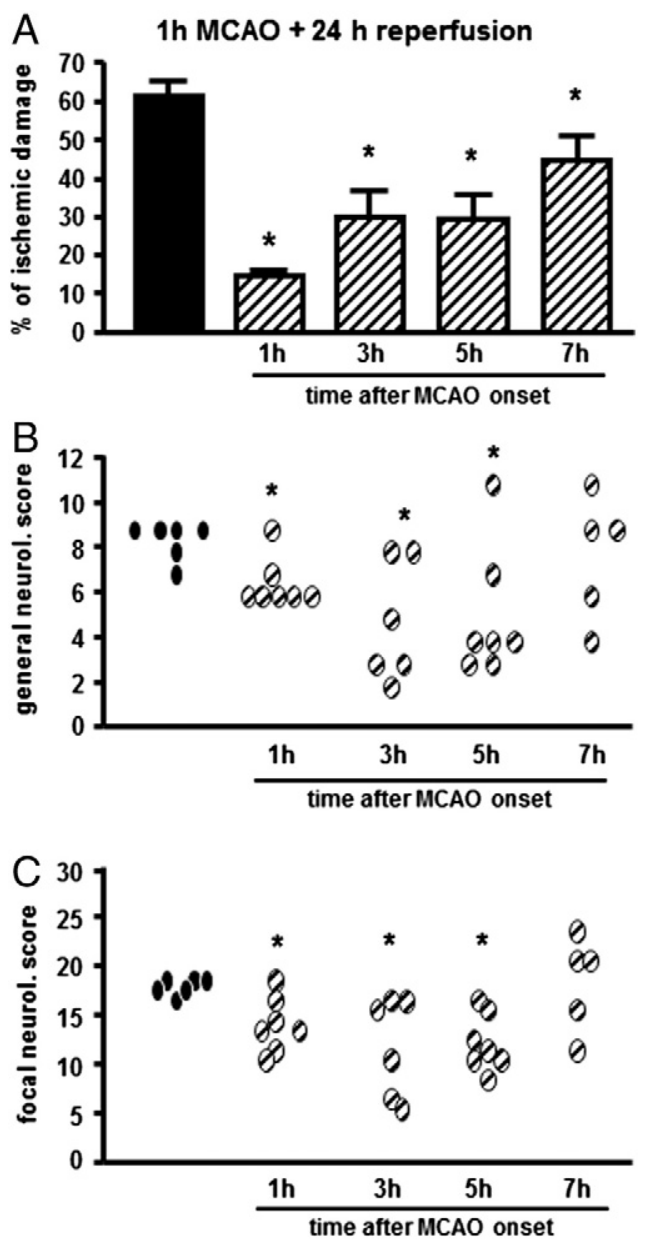

MCAO + vehicle

Z MCAO + resv. $(68 \mu \mathrm{g} / \mathrm{kg})+\mathrm{MS}-275(2 \mu \mathrm{g} / \mathrm{kg})$
A
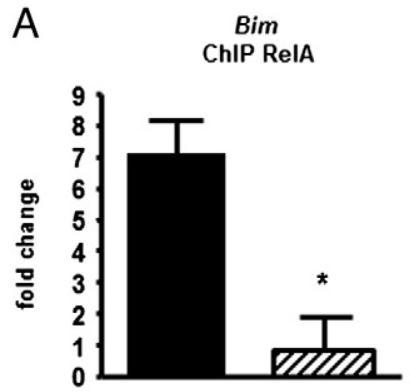

C

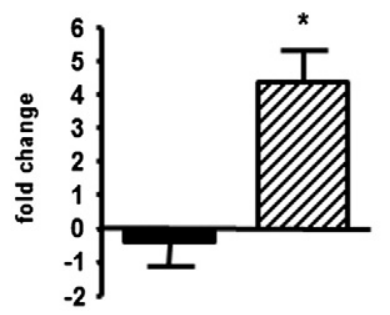

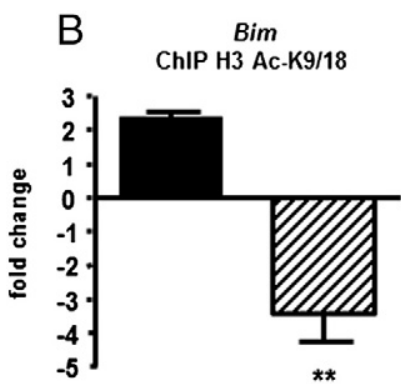

D $B c l-x_{L}$ ChIP H3 Ac-K9/18

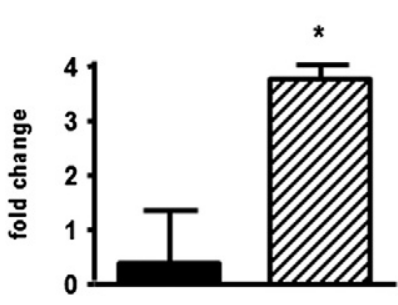

MCAO + vehicle

$\square$ MCAO + resv. $(68 \mu g / k g)+M S-275(2 \mu g / k g)$

Fig. 7. ChIP analysis of RelA and histone $\mathrm{H} 3$ acetylation (K9/18) on the pro-apoptotic Bim promoter (A and B) and the $B c l-x_{L}$ promoter (C and D). Treatment with MS-275 and resveratrol during 3 h-reperfusion significantly reduces both RelA binding and the acetylation of $\mathrm{H} 3$ histones associated with the Bim promoter, while increasing the recruitment of RelA and the acetylation of $\mathrm{H} 3$ histones associated with the $B c l-x_{L}$ promoter. Results are obtained by qRT-PCR analyses of Bim or $B c l-x_{L}$ promoter in immunoprecipitated DNA of brain tissue from mice exposed to MCAO and treated with vehicle or drug combination. Data are expressed as fold changes over values obtained in cortices from sham operated mice. Bars depict the mean \pm s.e.m. of three separate experiments run in triplicate ${ }^{*} p<0.05$ or ${ }^{* *} p<0.01$ versus the corresponding value.

already used in various clinical trials, decreases the infarct volume. Moreover, we demonstrate that MS-275 and resveratrol elicit a large synergistic effect when co-administered at sub-threshold doses.

Recent studies define histone acetylation as a master epigenetic mechanism in the regulation of gene expression, and enhanced histone acetylation patterns could be referred to as a diagnostic signature of ongoing active gene expression events (Boutillier et al., 2003; Gan et al., 2005; Lutz et al., 2000). In our cell-based model of brain ischemia, histone $\mathrm{H} 3$ acetylation at the $\mathrm{K} 9 / 18$ residues was drastically decreased, without evidence of a concomitant change in HDAC activities. This phenomenon has been reported to be a consequence of energy depletion in ischemic conditions leading to the cellular reduction of pyruvate dehydrogenase activity and decreased generation of acetyl-CoA. While a reduced availability of acetyl-CoA, the fundamental co-factor for HAT activity, can underlay the decreased levels of histone acetylation within the ischemic brain (Calvani and Arrigoni-Martelli, 1999; Martin et al., 2005), the pharmacological inhibition of HDACs during brain ischemia has been found to limit reduction of histone acetylation due to the decreased HAT activity (Faraco et al., 2006).

In neurons exposed to OGD, the deacetylation of total $\mathrm{H} 3$ histones highly correlated with both $\mathrm{H} 3$ deacetylation and RelA detachment occurring at the $B c l-x_{L}$ promoter, but not with $\mathrm{H} 3$ acetylation and RelA recruitment at the Bim promoter. This may suggest that NF- $\kappa$ B binding at the target promoter is the event guiding the promoter-specific histone acetylation.

In regards to the possible mechanisms involved in the synergistic neuroprotection, we found that only at the higher, $1 \mu \mathrm{M}$ concentration, 
MS-275 per se inhibited the HDAC activity and increased histone H3 acetylation. At $0.1 \mu \mathrm{M}$, MS-275 produced a lower HDAC inhibition, insufficient in increasing histone acetylation. As previously described, MS-275 provides selectivity for HDAC1, HDAC2 and HDAC3 (Khan et al., 2008), with higher potency for HDAC1 (IC50 of $\sim 0.2 \mu \mathrm{M}$ ), which display as a common target the entire $\mathrm{H} 3$ histone (Johnson et al., 2002). A decrease in general HDAC activity was also observed after application of either 3 or $30 \mu \mathrm{M}$ resveratrol, possibly because resveratrol can inhibit the HDAC6 isoform (Blackwell et al., 2008). Though, as H3 is not a target for the HDAC6 (Johnson et al., 2002), resveratrol did not modify per se the $\mathrm{H} 3$ acetylation, but produced a synergistic effect when associated with MS-275. To investigate metabolic changes possibly responsible for synergistic $\mathrm{H} 3$ acetylation, we evaluated the activity of the AMPK, a resveratrol target regulating the metabolic balance (Culmsee et al., 2001; Hawley et al., 2005; Turnley et al., 1999). While MS-275 appeared to be ineffective, resveratrol, even at the lower concentration, was able to induce AMPK phosphorylation at the T172 residue after OGD. AMPK function is recognized to activate many catabolic pathways that produce ATP and induce NARP to generate $\mathrm{NAD}^{+}$. It can be hypothesized that the metabolic improvement induced by resveratrol, via increased generation of acetyl-CoA in neuronal cells (Turnley et al., 1999), can sustain HAT activity, thus contributing to the observed recovery of $\mathrm{H} 3$ acetylation when co-administered with a low concentration of MS-275. Moreover, by providing NAD ${ }^{+}$, the fundamental co-factor for class III HDACs, AMPK has been found to indirectly corroborate sirtuin 1 activation by resveratrol (Ruderman et al., 2010) (Fig. 8).

Sirtuin 1 interacts with several proteins that are involved in key cellular processes, including mitochondrial biogenesis, autophagy and stress-induced responses. The biological effects of sirtuin 1 are mediated by its capability to deacetylate a wide range of transcriptional factors, such as peroxisome proliferator-activated receptor-c

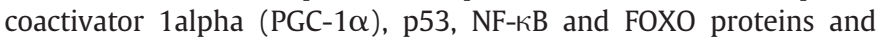

consequently regulate their activities (Wang et al., 2011). Our investigation does not rule out that other known targets of resveratrol/sirtuin 1 besides RelA acetylation may be involved in the pro-survival effect of the drug. Certainly, activation of the AMPK-sirtuin 1 pathway played an important role in the synergy of MS-275 and resveratrol. Either AMPK inhibition by compound $\mathrm{C}$ or sirtuin 1 inhibition by sirtinol completely abolished the neuroprotection induced by combined drugs in neurons exposed to OGD.

We evaluated the capability of MS-275 and resveratrol, used independently and in combination, to modulate RelA acetylation in neurons exposed to OGD. MS-275 at $0.1 \mu \mathrm{M}$ concentration, ineffective in restoring $\mathrm{H} 3$ acetylation, significantly increased the total RelA acetylation, suggesting that RelA is the primary target for HDACs, possibly HDAC1 (Khan et al., 2008), inhibited by MS-275. Resveratrol at $3 \mu \mathrm{M}$ did not affect per se the RelA acetylation but, when co-administered with MS-275, it caused deacetylation of RelA K310, a recognized target of sirtuin 1-deacetylase activity (Lanzillotta et al., 2010; Yeung et al., 2004). As a result of the MS-275 and resveratrol combination, the RelA general acetylation increased while the K310 acetylation decreased (Fig. 8). This global change in the status of RelA acetylation was not reproduced by the individual drugs, even when used at higher concentrations. These findings suggest that resveratrol-activated sirtuin 1 may display a higher affinity for RelA K310 when RelA is globally hyper-acetylated by MS-275. The exact mechanism responsible for increased affinity of sirtuin 1 to highly acetylated RelA remains to be elucidated.

The neuroprotection and anti-apoptotic transcription appeared to be closely related to the capability of the drug combination to restore the optimal RelA acetylation, thus reproducing pharmacologically what occurs in preconditioning ischemia (Lanzillotta et al., 2010; Raval et al., 2006). ChIP analysis revealed a strong decrease in RelA binding and $\mathrm{H} 3$ acetylation at the Bim promoter of neurons treated with MS-275 and resveratrol after the OGD. It suggests that the detachment of RelA

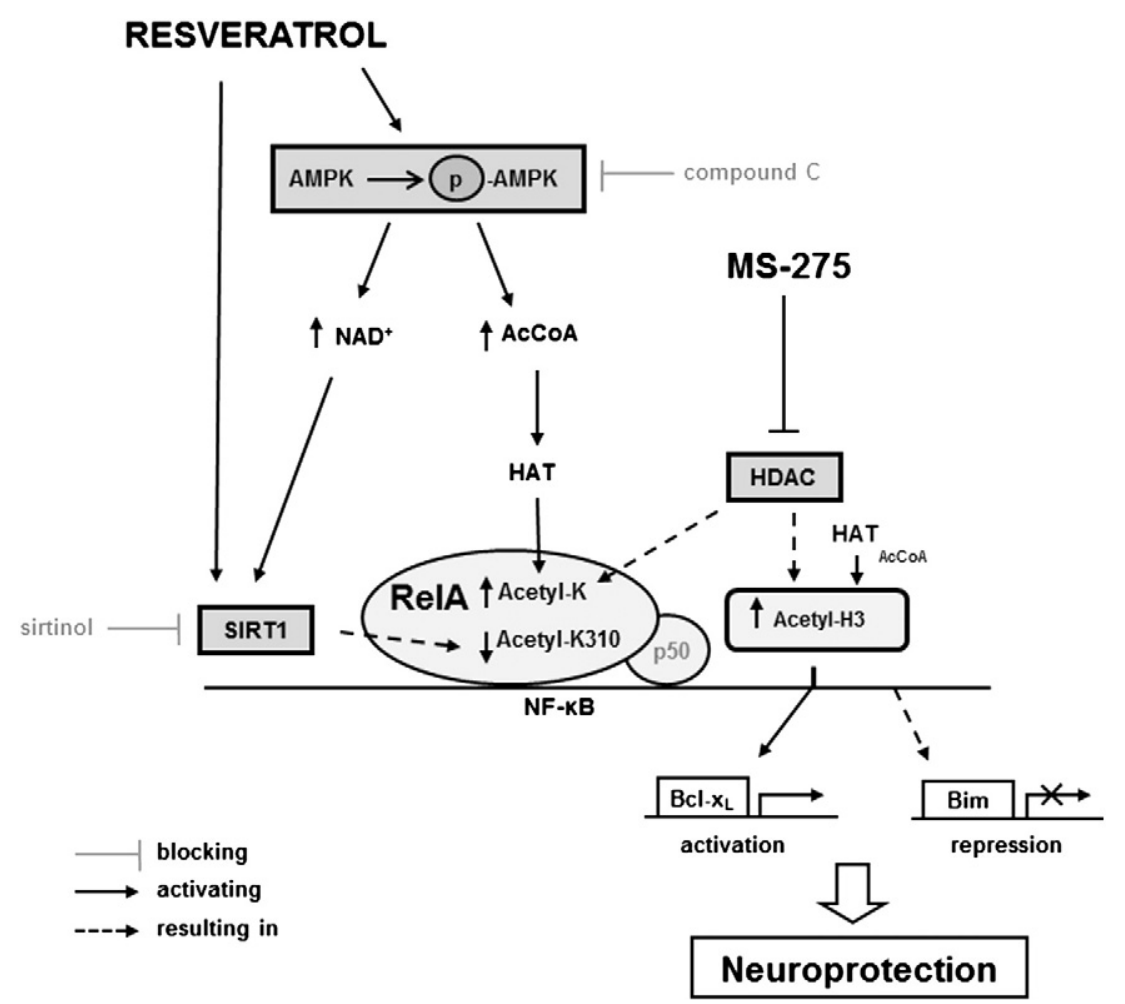

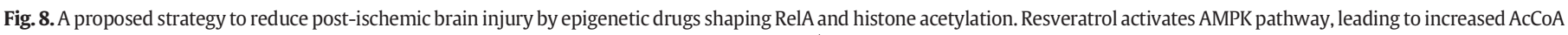

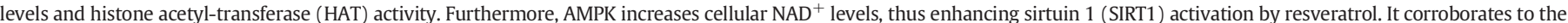

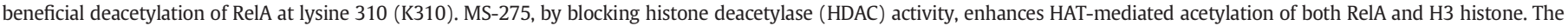

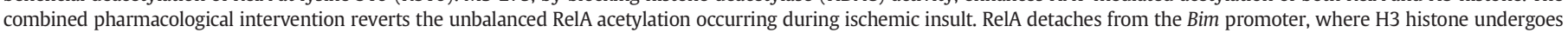
deacetylation, to repress pro-apoptotic Bim expression. Conversely, RelA binds the $B c l-x_{L}$ promoter and drives histone $\mathrm{H} 3$ acetylation to induce anti-apoptotic $B c l-x_{L}$ expression. 
from its $\kappa \mathrm{B}$-binding site may reduce both HAT recruitment at the transcriptional complex and acetylation of promoter associated-H3 histone.

Interestingly, the protective and transcriptional effects produced by resveratrol and MS-275 in cortical neurons exposed to OGD were entirely reproduced in the mouse MCAO model. The combination of the drugs, at inactive doses per se, elicited a synergistic effect that led to a higher reduction of brain damage than that produced by individual drugs at 100 -fold higher doses.

Pharmacokinetics studies reported short half-life for both drugs, approximately 15 min for resveratrol (Asensi et al., 2002) and $1 \mathrm{~h}$ for MS-275 (Ryan et al., 2005). Thus, the in vivo efficacy of such low doses highlights the still underestimated capability of resveratrol and MS-275 to reach injured brain and activate high-affinity mechanisms which can cooperate to afford neuroprotection. In this context, using 10-fold higher doses of drugs in combination did not improve the final beneficial effects. In mice subjected to MCAO, drugs administered in the reperfusion period significantly reduced cerebral infarct volume and limited the subsequent neuronal deficits. The treatment had a long-lasting efficacy as the beneficial effects were still evident $72 \mathrm{~h}$ after the injury. Furthermore, the treatment was efficacious even when administered $7 \mathrm{~h}$ after the ischemic onset.

Neuroprotection was associated with the capability of the drug combination to finally switch transcription from pro- to anti-apoptotic genes, as RelA binding shifted from the Bim to the $B c l-x_{L}$ promoter. Consequently, the acetylation of $\mathrm{H} 3$ histone associated with the Bim promoter decreased, while the $\mathrm{H} 3$ acetylation at the $B c l-x_{L}$ promoter increased (Fig. 8). These findings provide clear evidence that a pharmacological intervention affecting the epigenetic machinery regulating gene transcription can reduce post-ischemic brain damage.

\section{Conclusions}

The current findings show that aberrant RelA acetylation and histone deacetylation are both targeted by synergistic low doses of MS-275 and resveratrol after brain ischemia. The treatment is effective even after a delayed administration of $7 \mathrm{~h}$. This allows for a great timeframe for action to restore brain damages. The immediate hospitalization of patients with a cerebral ischemia is considered essential for the implementation of a therapy that can avoid the aggravation of symptoms and ameliorate the sequelae of stroke itself. The current evidence that neuroprotection and the reduction of neurological deficits are still achieved when the treatment is performed $7 \mathrm{~h}$ after the stroke onset place the efficacy of this strategy even beyond the time window for thrombolysis, suggesting that it may either implement or replace the use of tPA.

The preclinical results of the present study may be crucial to determine the translational implications in the intensive care of cerebral ischemia.

Supplementary data to this article can be found online at http:// dx.doi.org/10.1016/j.nbd.2012.08.018.

\section{Conflict of interest statement}

The authors declare no conflict of interest.

\section{Acknowledgments}

This work was supported by PRIN 2008 and the NEDD Project (CUP H81J09002660007), Regione Lombardia, Italy; Ricerca Sanitaria RF-FSL352059; Progetto Ordinario 2007; and PON_01602 Ricerca e Competitività 2007-2013. We thank Simone Di Giovanni for his advice and thoughtful discussion and Elisa Floriddia for her kind support and technical assistance in developing the ChIP protocol.

\section{References}

Agrawal, M., Kumar, V., Kashyap, M.P., Khanna, V.K., Randhawa, G.S., Pant, A.B., 2011 Ischemic insult induced apoptotic changes in PC12 cells: protection by trans resveratrol. Eur. J. Pharmacol. 666, 5-11.

Asensi, M., Medina, I., Ortega, A., Carretero, J., Baño, M.C., Obrador, E., et al., 2002. Inhibition of cancer growth by resveratrol is related to its low bioavailability. Free Radic. Biol. Med. 33, 387-398.

Ashburner, B.P., Westerheide, S.D., Baldwin Jr., A.S., 2001. The p65 (RelA) subunit of NF-kappaB interacts with the histone deacetylase (HDAC) corepressors HDAC1 and HDAC2 to negatively regulate gene expression. Mol. Cell. Biol. 21, 7065-7077.

Baur, J.A., Sinclair, D.A., 2006. Therapeutic potential of resveratrol: the in vivo evidence. Nat. Rev. Drug Discov. 5, 493-506.

Bederson, J.B., Pitts, L.H., Germano, S.M., Nishimura, M.C., Davis, R.L., Bartkowski, H.M. 1986. Evaluation of 2,3,5-triphenyltetrazolium chloride as a stain for detection and quantification of experimental cerebral infarction in rats. Stroke 17, 1304-1308.

Blackwell, L., Norris, J., Suto, C.M., Janzen, W.P., 2008. The use of diversity profiling to characterize chemical modulators of the histone deacetylases. Life Sci. 82, 1050-1058.

Boutillier, A.L., Trinh, E., Loeffler, J.P., 2003. Selective E2F-dependent gene transcription is controlled by histone deacetylase activity during neuronal apoptosis. J. Neurochem. 84, 814-828.

Bramlett, H.M., Dietrich, W.D., 2004. Pathophysiology of cerebral ischemia and brain trauma: similarities and differences. J. Cereb. Blood Flow Metab. 24, 133-150.

Calvani, M., Arrigoni-Martelli, E., 1999. Attenuation by acetyl-L-carnitine of neurologica damage and biochemical derangement following brain ischemia and reperfusion. Int. J. Tissue React. 21, 1-6.

Chen, L.F., Greene, W.C., 2004. Shaping the nuclear action of NF-kappaB. Nat. Rev. Mol. Cell Biol. 5, 392-401.

Clark, W.M., Lessov, N.S., Dixon, M.P., Eckenstein, F., 1997. Monofilament intraluminal middle cerebral artery occlusion in the mouse. Neurol. Res. 19, 641-648.

Culmsee, C., Monnig, J., Kemp, B.E., Mattson, M.P., 2001. AMP-activated protein kinase is highly expressed in neurons in the developing rat brain and promotes neuronal survival following glucose deprivation. J. Mol. Neurosci. 17, 45-58.

Fang, M.C., Cutler, D.M., Rosen, A.B., 2010. Trends in thrombolytic use for ischemic stroke in the United States. J. Hosp. Med. 5, 406-409.

Faraco, G., Pancani, T., Formentini, L., Mascagni, P., Fossati, G., Leoni, F., et al., 2006 Pharmacological inhibition of histone deacetylases by suberoylanilide hydroxamic acid specifically alters gene expression and reduces ischemic injury in the mouse brain. Mol. Pharmacol. 70, 1876-1884.

Gan, Y., Shen, Y.H., Wang, J., Wang, X., Utama, B., Wang, J., et al., 2005. Role of histone deacetylation in cell-specific expression of endothelial nitric-oxide synthase. J. Biol. Chem. 280, 16467-16475.

Haberland, M. Montgomery, R.L., Olson, E.N., 2009. The many roles of histone deacetylases in development and physiology: implications for disease and therapy Nat. Rev. Genet. 10, 32-42.

Hahnen, E., Hauke, J., Trankle, C., Eyupoglu, I.Y., Wirth, B., Blumcke, I., 2008. Histone deacetylase inhibitors: possible implications for neurodegenerative disorders. Expert Opin. Investig. Drugs 17, 169-184.

Hawley, S.A., Pan, D.A., Mustard, K.J., Ross, L., Bain, J., Edelman, A.M., et al., 2005 Calmodulin-dependent protein kinase kinase-beta is an alternative upstream kinase for AMP-activated protein kinase. Cell Metab. 2, 9-19.

Howitz, K.T., Bitterman, K.J., Cohen, H.Y., Lamming, D.W., Lavu, S., Wood, J.G., et al., 2003. Small molecule activators of sirtuins extend Saccharomyces cerevisiae lifespan. Nature 425, 191-196.

Inta, I., Paxian, S., Maegele, I., Zhang, W., Pizzi, M., Spano, P., et al., 2006. Bim and Noxa are candidates to mediate the deleterious effect of the NF-kappa B subunit RelA in cerebral ischemia. J. Neurosci. 26, 12896-12903.

Jin, K., Mao, X.O., Simon, R.P., Greenberg, D.A., 2001. Cyclic AMP response element binding protein (CREB) and CREB binding protein (CBP) in global cerebral ischemia. J. Mol. Neurosci. 16, 49-56.

Johnson, C.A., White, D.A., Lavender, J.S., O'Neill, L.P., Turner, B.M., 2002. Human class I histone deacetylase complexes show enhanced catalytic activity in the presence of ATP and co-immunoprecipitate with the ATP-dependent chaperone protein Hsp70. J. Biol. Chem. 277, 9590-9597.

Khan, N., Jeffers, M., Kumar, S., Hackett, C., Boldog, F., Khramtsov, N., et al., 2008. Determination of the class and isoform selectivity of small-molecule histone deacetylase inhibitors. Biochem. J. 409, 581-589.

Kim, H.J., Rowe, M., Ren, M., Hong, J.S., Chen, P.S., Chuang, D.M., 2007. Histone deacetylase inhibitors exhibit anti-inflammatory and neuroprotective effects in a rat permanent ischemic model of stroke: multiple mechanisms of action. J. Pharmacol. Exp. Ther. 321, 892-901.

Kim, H.J., Leeds, P., Chuang, D.M., 2009. The HDAC inhibitor, sodium butyrate, stimulates neurogenesis in the ischemic brain. J. Neurochem. 110, 1226-1240.

Langley, B., D'Annibale, M.A., Suh, K., Ayoub, I., Tolhurst, A., Bastan, B., et al., 2008. Pulse inhibition of histone deacetylases induces complete resistance to oxidative death in cortical neurons without toxicity and reveals a role for cytoplasmic p21(waf1/cip1) in cell cycle-independent neuroprotection. J. Neurosci. 28, 163-176.

Lanzillotta, A Sarnico, I. Ingrassia, R., Boroni, F., Branca, C., Benarese, M., et al, 2010. The acetylation of RelA in Lys310 dictates the NF-kB-dependent response in post-ischemic injury. Cell Death Dis. 1, e96.

Lees, K.R., Bluhmki, E., von Kummer, R., Brott, T.G., Toni, D., Grotta, J.C., et al., 2010. Time to treatment with intravenous alteplase and outcome in stroke: an updated pooled analysis of ECASS, ATLANTIS, NINDS, and EPITHET trials. Lancet 375, 1695-1703.

Leker, R.R., Shohami, E., Constantini, S., 2002. Experimental models of head trauma. Acta Neurochir. Suppl. 83, 49-54. 
Li, H., Yan, Z., Zhu, J., Yang, J., He, J., 2011. Neuroprotective effects of resveratrol on ischemic injury mediated by improving brain energy metabolism and alleviating oxidative stress in rats. Neuropharmacology 60, 252-258.

Lloyd-Jones, D., Adams, R.J., Brown, T.M., Carnethon, M., Dai, S., De Simone, G., et al. 2010. Executive summary: heart disease and stroke statistics - 2010 update: a report from the American Heart Association. Circulation 121, 948-954.

Lutz, M., Burke, L.J., Barreto, G., Goeman, F., Greb, H., Arnold, R., et al., 2000. Transcriptional repression by the insulator protein CTCF involves histone deacetylases. Nucleic Acids Res. 28, 1707-1713.

Martin, E., Rosenthal, R.E., Fiskum, G., 2005. Pyruvate dehydrogenase complex: metabolic link to ischemic brain injury and target of oxidative stress. J. Neurosci. Res. 79, 240-247.

Morris, K.C., Lin, H.W., Thompson, J.W., Perez-Pinzon, M.A., 2011. Pathways for ischemic cytoprotection: role of sirtuins in caloric restriction, resveratrol, and ischemic preconditioning. J. Cereb. Blood Flow Metab. 31, 1003-1019.

Pignataro, G., Meller, R, Inoue, K, Ordonez, A.N., Ashley, M.D., Xiong Z et al, 2008. In vivo and in vitro characterization of a novel neuroprotective strategy for stroke: ischemic postconditioning. J. Cereb. Blood Flow Metab. 28, 232-241.

Pizzi, M., Sarnico, I., Boroni, F., Benarese, M., Steimberg, N., Mazzoleni, G., et al., 2005. NF-kappaB factor c-Rel mediates neuroprotection elicited by mGlu5 receptor agonists against amyloid beta-peptide toxicity. Cell Death Differ. 12, 761-772.

Raval, A.P., Dave, K.R., Pérez-Pinzón, M.A., 2006. Resveratrol mimics ischemic preconditioning in the brain. J. Cereb. Blood Flow Metab. 26, 1141-1147.

Ren, J., Fan, C., Chen, N., Huang, J., Yang, Q., 2011. Resveratrol pretreatment attenuates cerebral ischemic injury by upregulating expression of transcription factor Nrf2 and $\mathrm{HO}-1$ in rats. Neurochem. Res. 36, 2352-2362.

Robb, E.L., Stuart, J.A., 2010. trans-Resveratrol as a neuroprotectant. Molecules 15, 1196-1212.

Rocha, S., Campbell, K.J., Perkins, N.D., 2003. p53- and Mdm2-independent repression of NF-kappa B transactivation by the ARF tumor suppressor. Mol. Cell 12, 15-25.

Rouaux, C., Jokic, N., Mbebi, C., Boutillier, S., Loeffler, J.P., Boutillier, A.L., 2003. Critical loss of $\mathrm{CBP} / \mathrm{p} 300$ histone acetylase activity by caspase- 6 during neurodegeneration. EMBO J. 22, 6537-6549.
Ruderman, N.B., Xu, X.J., Nelson, L., Cacicedo, J.M., Saha, A.K., Lan, F., et al., 2010. AMPK and SIRT1: a long-standing partnership? Am. J. Physiol. Endocrinol. Metab. 298, E751-E760.

Ryan, Q.C., Headlee, D., Acharya, M., Sparreboom, A., Trepel, J.B., Ye, J., et al., 2005. Phase I and pharmacokinetic study of MS-275, a histone deacetylase inhibitor, in patients with advanced and refractory solid tumors or lymphoma. J. Clin. Oncol. 23, 3912-3922.

Sarnico, I. Lanzillotta, A, Boroni, F., Benarese, M., Alghisi, M., Schwaninger, M., et al. 2009. NF-kappaB p50/RelA and c-Rel-containing dimers: opposite regulators of neuron vulnerability to ischaemia. J. Neurochem. 108, 475-485.

Shein, N.A., Shohami, E., 2011. Histone deacetylase inhibitors as therapeutic agents for acute central nervous system injuries. Mol. Med. 17, 448-456.

Simonini, M.V., Camargo, L.M., Dong, E., Maloku, E., Veldic, M., Costa, E., et al., 2006. The benzamide MS-275 is a potent, long-lasting brain region-selective inhibitor of histone deacetylases. Proc. Natl. Acad. Sci. U. S. A. 103, 1587-1592.

Smoliga, J.M., Baur, J.A., Hausenblas, H.A., 2011. Resveratrol and health - a comprehensive review of human clinical trials. Mol. Nutr. Food Res. 55, 1129-1141.

Sweatt, J.D., 2009. Experience-dependent epigenetic modifications in the CNS. Biol. Psychiatry 65, 191-197.

Tan, J., Cang, S., Ma, Y., Petrillo, R.L., Liu, D., 2010. Novel histone deacetylase inhibitors in clinical trials as anti-cancer agents. J. Hematol. Oncol. 3, 5.

Turnley, A.M., Stapleton, D., Mann, R.J., Witters, L.A., Kemp, B.E., Bartlett, P.F., 1999. Cellular distribution and developmental expression of AMP-activated protein kinase isoforms in mouse central nervous system. J. Neurochem. 72, 1707-1716.

Wang, Y., Liang, Y., Vanhoutte, P.M., 2011. SIRT1 and AMPK in regulating mammalian senescence: a critical review and a working model. FEBS Lett. 585, 986-994.

Yeung, F., Hoberg, J.E., Ramsey, C.S., Keller, M.D., Jones, D.R., Frye, R.A., et al., 2004. Modulation of NF-kappaB-dependent transcription and cell survival by the SIRT1 deacetylase. EMBO J. 23, 2369-2380.

Yu, W., Fu, Y.C., Wang, W., 2012. Cellular and molecular effects of resveratrol in health and disease. J. Cell. Biochem. 113, 752-759. 